\title{
Signatures of Dirac Cones in a DMRG Study of the Kagome Heisenberg Model
}

\author{
Yin-Chen He, ${ }^{1,2,3}$ Michael P. Zaletel, ${ }^{4,3,6}$ Masaki Oshikawa, ${ }^{5,3}$ and Frank Pollmann ${ }^{1,3,7}$ \\ ${ }^{1}$ Max-Planck-Institut für Physik komplexer Systeme, Nöthnitzer Straße 38, 01187 Dresden, Germany \\ ${ }^{2}$ Department of Physics, Harvard University, Cambridge, Massachusetts 02138, USA \\ ${ }^{3}$ Kavli Institute for Theoretical Physics, University of California, Santa Barbara, California 93106, USA \\ ${ }^{4}$ Station Q, Microsoft Research, Santa Barbara, California 93106, USA \\ ${ }^{5}$ Institute for Solid State Physics, University of Tokyo, Kashiwa 277-8581, Japan \\ ${ }^{6}$ Department of Physics, Princeton University, Princeton, New Jersey 08540, USA \\ ${ }^{7}$ Technische Universität München, Physics Department T42, 85747 Garching, Germany \\ (Received 30 November 2016; revised manuscript received 28 April 2017; published 28 July 2017)
}

\begin{abstract}
The antiferromagnetic spin-1/2 Heisenberg model on a kagome lattice is one of the most paradigmatic models in the context of spin liquids, yet the precise nature of its ground state is not understood. We use large-scale density matrix renormalization group simulations (DMRG) on infinitely long cylinders and find indications for the formation of a gapless Dirac spin liquid. First, we use adiabatic flux insertion to demonstrate that the spin gap is much smaller than estimated from previous DMRG simulation. Second, we find that the momentum-dependent excitation spectrum, as extracted from the DMRG transfer matrix, exhibits Dirac cones that match those of a $\pi$-flux free-fermion model [the parton mean-field ansatz of a $U(1)$ Dirac spin liquid].
\end{abstract}

DOI: 10.1103/PhysRevX.7.031020

Subject Areas: Computational Physics, Condensed Matter Physics, Strongly Correlated Materials

\section{INTRODUCTION}

Understanding the ground state of the antiferromagnetic spin-1/2 Heisenberg model on a kagome lattice (KAH) has proved to be one of the most vexed issues in quantum magnetism [1-24]. The KAH is one of the simplest models with strong frustration and is a reasonable starting point for understanding various layered magnets such as Herbertsmithite [25,26]. The possibility of a quantum spin liquid (QSL) [27] on the KAH was proposed more than two decades ago [1] and was more recently confirmed numerically through density matrix renormalization group (DMRG) simulations [8]. However, there is a multitude of possible QSLs $[28,29]$, and despite tremendous efforts, the precise nature of the QSL in the KAH remains unknown. Thus, we refer to it simply as the kagome spin liquid.

Currently, the most promising candidates are the gapped $Z_{2}$ spin liquid [1,30-32] $\left(Z_{2}\right.$ SL) and the gapless $U(1)$ Dirac spin liquid (DSL) [3]. Recent DMRG studies appear to support a gapped $Z_{2}$ SL scenario $[8,11,12]$. However, characteristic properties of the gapped $Z_{2} S L$, e.g., fourfold topological degeneracy on a torus and fractional statistics of spinons (e.g., through modular matrix [33-36]), have

Published by the American Physical Society under the terms of the Creative Commons Attribution 4.0 International license. Further distribution of this work must maintain attribution to the author(s) and the published article's title, journal citation, and DOI. not been observed. On the other hand, there are several indications favoring a DSL over a $Z_{2}$ SL. First, extensive variational Monte Carlo studies suggest a DSL [5,14-17]. Second, the kagome spin liquid was found to be proximate to a chiral spin liquid stabilized by the addition of longerranged spin exchange interactions [37,38], with indications that the transition between them is continuous [19]. While there is no known theory that could describe a continuous transition between a $Z_{2}$ SL and the chiral spin liquid $[39,40]$, such a transition occurs naturally if the kagome spin liquid is a DSL. Third, a recent theoretical work [41] suggests that a DSL is a natural possibility by investigating a lattice gauge theory formulation $[42,43]$ of the easy-axis (XXZ) kagome model $[19,20]$.

Most of the early experimental studies on candidate materials, such as Herbertsmithite, suggested a gapless spin-liquid scenario based on spin susceptibility and specific heat measurements [25]. However, the effective model for those materials is thought to be more complicated than the $\mathrm{KAH}$, and it was difficult to determine if these gapless signatures were intrinsic properties of the kagome spin liquid or were due to magnetic impurities. A recent NMR study of Herbertsmithite found evidence for a finite spin gap $(0.03 J-0.07 J)$ by using the Knight shift to filter out impurity contributions [44].

In this paper, we revisit the kagome spin-liquid problem using the DMRG [45-47] method, which remains one of the most unbiased and powerful numerical methods to deal with this problem. We systematically investigate the energy gap and excitation spectrum of the kagome spin-liquid 
phase by using extensions to the previously implemented algorithms: (i) We provide new insight into the heavily debated spin gap issue of the KAH by computing its dependence on boundary conditions and show that the spin gap from our DMRG simulations is consistent with a gapless QSL (e.g., DSL). (ii) We obtain the momentumresolved spectrum of correlation lengths of the KAH, which is closely related to the excitation spectrum [48]. In particular, this spectrum shows signatures of Dirac cones at the locations expected for a gapless DSL [3]. We emphasize that these signatures are seen in the same spin-liquid phase that was reported in previous DMRG simulations $[8,11]$, not a different, competing phase. The method we use here can also be directly applied to explore QSL phases in other lattice models.

The paper is organized as follows. We begin by reviewing some promising spin-liquid candidates and previous DMRG studies in Sec. II. We then discuss the expected behavior of various QSLs when placed on the cylinder geometry in Sec. III. We present our numerical DMRG data in Sec. IV. First, we show that the spin gap drops significantly compared to previously reported values as we twist the boundary conditions. Then, we extract the momentum-dependent excitation spectrum from the DMRG transfer matrix. The triplet excitations reveal a Dirac cone structure, with the Dirac point located at the $M$ point of the Brillouin zone, as expected for a $\pi$-flux DSL. We finally conclude with a summary and discussion in Sec. V.

\section{BRIEF REVIEW: KAGOME SPIN LIQUIDS}

\section{A. Previous DMRG studies}

Yan et al. [8] performed an extensive DMRG study of the kagome Heisenberg model on cylinders with circumference sizes from $L_{y}=2$ (e.g., YC4) to $L_{y}=6$ (e.g., YC12) unit cells. Most importantly, it was found that the ground state is a symmetric spin-liquid state that has a much lower energy than competing valence bond crystal states $[4,7]$. By performing a careful finite-size scaling analysis on different geometries, an energy per site of $E_{0}=-0.4379(3)$ was estimated. Several observations were made regarding the nature of the spin-liquid state: (i) The ground state is a spin singlet protected by a small spin gap to the lowest-lying spin-1 state. A spin gap $\Delta_{S=1}=0.125(9) J$, with $J$ being the strength of the exchange interaction, was found for the $L_{y}=6$ (unit cells) cylinder (XC12-2). This gap is smaller than the gap of $\Delta_{S=1}=0.164 \mathrm{~J}$ extracted from earlier exact diagonalization studies of a 36-site cluster [49]. (ii) The singlet gap, separating the ground state from the lowest spin-0 excited state, is estimated to be much smaller, $\Delta_{S=0}=0.054(9)$ for the $L_{y}=6$ (unit cells) cylinder (XC12-2). This differs strongly from the exact diagonalization results that estimate the singlet gap to be less than $0.01 J$ [49]. The difference is attributed to strong finitesize effects for the small clusters used for the exact diagonalization studies. (iii) The ground state is found to have a very short correlation length. The findings of Yan et al. were further corroborated by the $S U(2)$-invariant DMRG study of Depenbrock et al. [11]. It was further argued based on the entanglement properties that the spin liquid is likely a $Z_{2}$ spin liquid $[11,12]$.

\section{B. Parton constructions for kagome spin liquids}

For the discussion that follows, it will prove very useful to have a picture of the competing phases within the language of the fermionic parton construction. Here, we briefly review the parton construction for a $2 \mathrm{D}$ plane $[28,29,50]$; in Sec. III, we discuss the novel phenomena that arise when a QSL is wrapped onto a cylinder.

In the parton construction, the physical spin operator is expressed as a bilinear of a fictitious $S=1 / 2$ fermion, $\hat{\mathbf{S}}_{i}=\sum_{\sigma, \sigma^{\prime}} f_{i, \sigma^{\dagger}}^{\dagger} \mathbf{S}_{\sigma, \sigma^{\prime}} f_{i, \sigma^{\prime}}$. In order to reproduce the correct properties for $\hat{\mathbf{S}}_{i}$, one enforces the constraint $1=\sum_{\sigma} f_{i, \sigma}^{\dagger} f_{i, \sigma}$. This introduces an extensive redundancy: For example, the spin operator is left invariant under $U(1)$ "gauge transformations" $f_{i, \sigma} \rightarrow e^{i \phi_{i}} f_{i, \sigma}$ [in fact, there is actually a larger $S U(2)$ redundancy]. In the resulting effective field theory, the fermionic partons are coupled to an emergent gauge field that both enforces the constraint and implements the microscopic redundancy as a gauge invariance.

Rather than elaborating on the field theory, we use the parton construction as a variational ansatz for the ground state and its low-lying excitations. Letting $|\mathrm{MF}\rangle$ be an ansatz free-fermion wave function for the $f$, we can obtain a spin$1 / 2$ wave function by projecting onto a single occupancy,

$$
|\Psi\rangle=\prod_{i} n_{i}\left(2-n_{i}\right)|\mathrm{MF}\rangle=\mathcal{P}_{G}|\mathrm{MF}\rangle .
$$

Various nontrivial spin-liquid phases result by choosing $|\mathrm{MF}\rangle$ to be the ground state of certain free-fermion $H_{\mathrm{f}}$, as will be discussed below.

The parton picture simultaneously suggests an ansatz for the excitations. If $\gamma_{\sigma}$ is a low-lying excitation of $H_{\mathrm{f}}$, with $\sigma=\uparrow / \downarrow$, we can obtain a corresponding ansatz for a low-lying excitation of the spin system,

$$
\left|\gamma_{\sigma}\right\rangle=\mathcal{P}_{G} \gamma_{\sigma}^{\dagger}|\mathrm{MF}\rangle
$$

This excitation is called a fermionic "spinon" excitation since it carries an $\mathrm{S}=1 / 2$ moment. Consequently, the spinon is a topological excitation; e.g., it cannot be made by acting with the local $\mathbf{S}_{i}$ operators in some patch. Triplet excitations are obtained from the two-spinon states. If the mean-field description is well behaved, the energy of the ansatz excitation $\left|\gamma_{\sigma}\right\rangle$ should have some qualitative relation to the energy of $\gamma$ in $H_{\mathrm{f}}$ : for example, the location of band minima or gapless points.

We now turn to the free-fermion ansatz $H_{\mathrm{f}}$ thought to be relevant for the kagome spin liquid. 


\section{Gapped $\mathbb{Z}_{2}$ spin liquid}

We obtain a gapped $Z_{2}$ spin liquid $[1,30,31]$ by supposing that the partons form a Bardeen-CooperSchrieffer (BCS) superconductor: $H_{\mathrm{f}}=-\sum_{\langle i, j\rangle, \sigma} t_{i, j} f_{i \sigma}^{\dagger} f_{j \sigma}+$ $\Delta_{i j} f_{i \uparrow} f_{i \downarrow}+$ H.c. $-\mu N$. Various choices of sign structures for the $t, \Delta$ (corresponding, for example, to patterns of $\pi$ flux through plaquettes) in fact lead to eight known gapped $\mathrm{Z}_{2}$-SLs, which differ in how the crystal symmetries act on the spinon excitations [1,51-54]. In all eight cases, the Bogoliubov de Gennes (BdG) particles $\gamma_{\sigma}$ are gapped, leading to a spin gap. There is also a second type of excitation in a $\mathbb{Z}_{2}$ spin liquid: a $\pi$ flux of the emergent gauge field, which is coupled to the partons (the other gauge excitations are at very high energies because of the Higgs mechanism). An ansatz for this $\pi$ flux (a.k.a. "vison") excitation is given by appropriately modifying the pairing $t_{i j} \rightarrow-t_{i j}, \Delta_{i j} \rightarrow-\Delta_{i j}$ along a semi-infinite line to mimic a $\pi$ flux piercing the plane at the endpoint, finding the resulting free-fermion ground state, and Gutzwiller projecting. The $\pi$ flux is also a topological excitation, but it does not carry spin. The existence of the $\pi$ flux leads to important phenomena on the cylinder, which we discuss later.

\section{2. $U(1)-D i r a c$ spin liquid}

In the $U(1)$-Dirac spin liquid, we choose a hopping-only ansatz [3,5,14-17]:

$$
H_{\mathrm{f}}=-\sum_{\langle i, j\rangle, \sigma} t_{i, j} f_{i \sigma}^{\dagger} f_{j \sigma}+\text { H.c. }-\mu N .
$$

The uniform nearest-neighbor ansatz $t_{i j}=1$ is a poor choice for the kagome model since it leads to a flat valence band: With an odd number of sites in the unit cell at a density of $n=1$ per site, this band would be half full. Instead, one considers the hopping pattern shown in Fig. 1(b), which has a $\pi$ flux piercing the hexagons. This $\pi$-flux ansatz results in a halved magnetic Brillouin zone with two Dirac cones at momenta $Q=(\pi / 2, \pi / 2), Q^{\prime}=(-\pi / 2,-\pi / 2)$. Combined with spin, there are $N_{f}=4$ gapless Dirac cones and hence a vanishing spin gap. Since the emergent gauge field is not "Higgsed," there are also low-energy gauge fluctuations, and the resulting effective theory is essentially $N_{f}=4 \mathrm{QED}_{3}$.

\section{Chiral spin liquid}

The chiral spin liquid is also a hopping-only ansatz $[3,55,56]$, but we choose the $t_{i j}$ to have phases such that the occupied bands have a total Chern number of $C=1$ per spin species. From the point of view of the DSL, it is obtained by turning on the same-sign mass term, $\bar{\psi}_{Q, \sigma} \psi_{Q, \sigma}$, on all $N_{f}=4$ Dirac cones. By choosing $C=1$, the ansatz breaks both time-reversal $T$ and reflection $P$, though their combination $P T$ is preserved. Generically, this leads to a nonvanishing expectation value for the chiral order

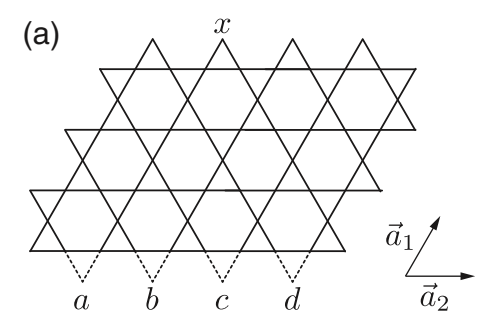

(b)
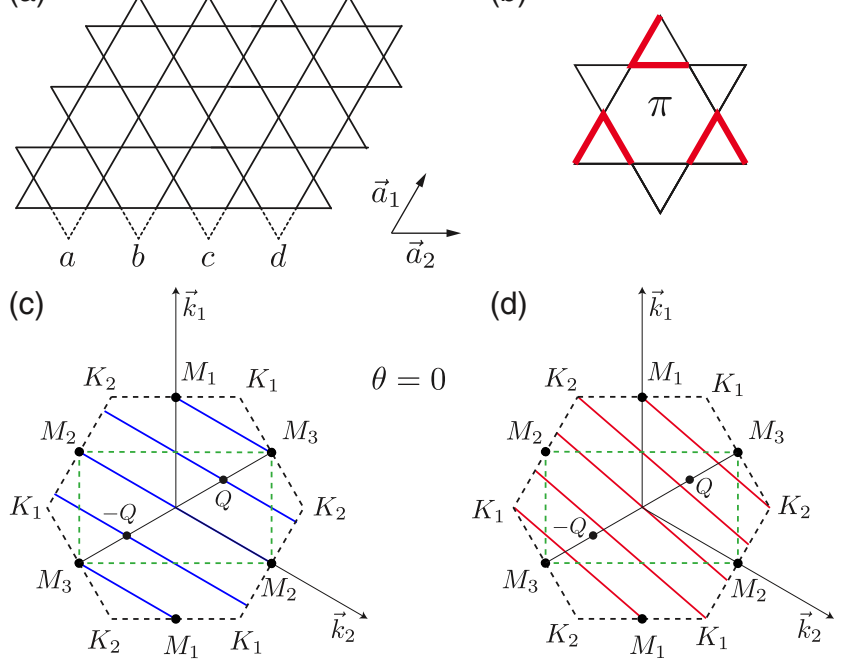

FIG. 1. (a) Illustration of the kagome lattice with a width of $L_{y}=4$ unit cells. The different geometries, YC8-0, YC8-2, YC84, and YC8-6, correspond to identifying the site $x$ with the site $a$, $b, c$, or $d$, respectively. (b) The $\pi$-flux state, where each hexagon has $\pi$ flux. We chose a specific gauge, in which the red bond has $s_{i j}=-1$, and the black bond has $s_{i j}=1$. The different geometries [here (c) YC8-0 and (d) YC8-2] correspond to different ways of cutting the Brillouin zone (shown as blue and red lines). The $M$ points are [labeled by $\left.\left(k_{1}, k_{2}\right)\right], M_{1}=(\pi, 0), M_{2}=(0, \pi)$, and $M_{3}=(\pi, \pi)$. The DSL has a halved magnetic Brillouin zone (dashed line) because it is a $\pi$-flux ansatz. The two Dirac points of the DSL are at $\pm Q$, and we refer to this as $Q, Q^{\prime}$ in the text. Note the blue and red lines represent cuts in the original Brillouin zone; the allowed momenta of the fractionalized spinons form similar cuts through the magnetic Brillouin zone, but the cuts are displaced according to the spin flux $\theta$ and the emergent gauge flux $\phi$, as we explain in Sec. III C.

parameter $\vec{S}_{i}\left(\vec{S}_{j} \times \vec{S}_{k}\right)$, where $i, j, k$ are three nearby sites. Since the Chern band is gapped, there is a finite energy cost for spinon excitations, and the emergent gauge field is gapped by the effective Chern-Simons term. The ChernSimons term leads to a spin-Hall coefficient of $\sigma_{H}^{(s)}=\frac{1}{2}$.

Note that the Dirac-SL can be considered the "parent state" for many of these spin liquids: The $\mathrm{Z}_{2}$ gapped states are obtained by turning on some superconducting terms, while the CSL arises if a staggered $B$ field spontaneously forms in addition to the $\pi$ flux. This picture gives a natural scenario for continuous phase transitions into these neighboring phases.

\section{SPIN LIQUIDS ON A CYLINDER}

Before providing numerical DMRG results for the kagome spin liquid, it is worthwhile to pause and consider what one should expect for a spin liquid in the DMRG simulation, which compactifies the kagome lattice into a cylinder. Since spin liquids are topological phases, they display a number of subtle features on a cylinder. 
(a)

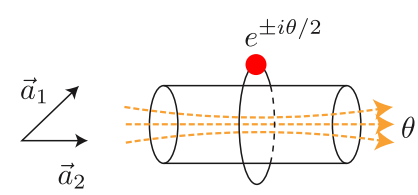

(c)



$\psi_{1} \mathrm{APBC}$

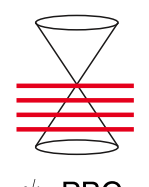

$\psi_{2} \mathrm{PBC}$

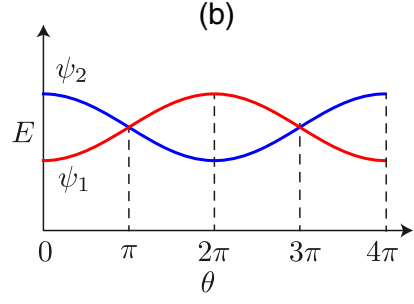

(d)

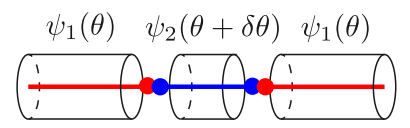

FIG. 2. Diagram for a spin liquid under the twist. (a) The spinon feels half-twist boundary or flux due to the fractionalization. (b) The response of the energy under the twist. (c) Two topological sectors of a DSL, with one gapped sector and one gapless sector. (d) The collapse of the twist simulation will have a quasiparticle emerging as the domain wall.

\section{A. Kagome cylinder geometries}

A cylinder is defined by identifying sites that differ by some $\vec{r}$, which defines the different compactifications. We work with the YC2n-2m cylinders, where $\vec{x}$ is identified with $\vec{x}+n \vec{a}_{1}-m \vec{a}_{2}$ and $\vec{a}_{1}, \vec{a}_{2}$ are the kagome Bravais vectors defined in Fig. 1(a). We have $n>m$, so we call $L_{y}=n$ the "circumference" of the cylinder, while $m$ amounts to a shift of the most naive identification $m=0$. We note that $\mathrm{YC} 2 \mathrm{n}-0$ and $\mathrm{YC} 2 \mathrm{n}-\mathrm{n}$ are actually the $\mathrm{YC} 2 \mathrm{n}$ and $\mathrm{XC} 2 \mathrm{n}$ geometries introduced in Ref. [8].

Since one direction of the system is compactified, the momentum along that direction is discretized, specifically

$$
n k_{1}+m k_{2}=0, \quad \bmod 2 \pi .
$$

Note that the other direction is infinite, so $k_{1}$ and $k_{2}$ can take continuous values so long as they satisfy the above relation. The different geometries (different $n$ and $m$ ) thus provide different cuts through the Brillouin zone, as shown in Figs. 1(c) and 1(d).

A second parameter that one can tune is the boundary condition $\theta$ of the spins around the circumference, the same way one would measure spin stiffness. We obtain a twist boundary condition by modifying Heisenberg coupling according to $S_{x, y}^{+} S_{x^{\prime}, y+1}^{-} \rightarrow e^{i \theta / L_{y}} S_{x, y}^{+} S_{x^{\prime}, y+1}^{-}$. By analogy to flux threading, we call $\theta$ the spin flux in the cylinder, as shown in Fig. 2(a).

\section{B. Gapped spin liquid on a cylinder}

Gapped spin liquids have fractionalized quasiparticles that have nontrivial statistics (e.g., the fermionic spinon). A direct consequence of such fractionalization is the topological degeneracy on a torus; There is one ground state per quasiparticle type [30,57]. Precisely the same

degeneracy arises for a long (infinite) cylinder-one can picture the ends of the cylinder as identified into a torus at infinity. The energy splitting between these "topological sectors" is exponentially small in the circumference.

In the $\mathrm{Z}_{2}-\mathrm{SL}$, the topological degeneracy can be understood in terms of the boundary conditions of the fermionic partons. While the boundary conditions of the spins are set by $\theta$, the fermionic spinons are coupled to an emergent gauge field, and the flux " $\phi$ " of this gauge field through the cylinder effectively changes their boundary conditions. In a superconductor, the $\pi$ flux is invisible to the condensate, so periodic ( $\mathrm{PBC}$ ) and antiperiodic (APBC) will give nearly degenerate energies - the splitting should decay exponentially in the cylinder circumference vs coherence length.

There is an interesting interplay between the topological degeneracy and spin flux $\theta$. Spin rotations $S_{i}^{ \pm} \rightarrow e^{ \pm i \Omega} S_{i}^{ \pm}$are implemented on the partons as $f_{i, \sigma} \rightarrow e^{i \Omega \sigma / 2} f_{i, \sigma}$, where $\sigma=$ \pm 1 corresponds to spin-up and spin-down components (the eigenvalue of $2 S^{z}$ ). As a consequence, the spin flux $\theta$ will change the boundary conditions of the up or down partons by $\pm \theta / 2$. A superconductor has no spin stiffness, so the change in the energy will again be exponentially small in the circumference of the cylinder. However, at $\theta=2 \pi, H_{\mathrm{f}}$ [in Eq. (3)] does not return to itself: Because they carry halfinteger spin, the boundary conditions for the $f_{\sigma}$ have been changed by $\pm 2 \pi / 2 \equiv \pi$. Equivalently, threading $2 \pi$-spin flux adiabatically exchanges between the PBC and APBC topological sectors, as shown in Fig. 2(b). Note that the location of the crossing is pinned to $\theta=\pi$ by time reversal, where it acts by exchanging the two topological sectors.

In summary, there is a striking signature of a gapped $\mathbb{Z}_{2}$ spin liquid: As spin flux is inserted, there should be an exponentially small change $\left(L_{x} \mathcal{O}\left[e^{-L_{\text {cyl }} / \xi}\right]\right)$ in the groundstate energy, and at $\theta=2 \pi$, the ground state should not return to itself but instead should thread the emergent $\pi$ flux that corresponds to the other topological degenerate ground state $[58,59]$. Furthermore, with those topological degenerate ground states, one can also calculate the modular matrix that fully characterizes the topological order of a gapped SL [35,36].

\section{Dirac spin liquid on a cylinder}

Like the $\mathbb{Z}_{2}$-SL, the DSL has an emergent gauge field that can dynamically change the boundary conditions of spinons (including either $\mathrm{PBC}$ or $\mathrm{APBC}$ ). Those $\mathrm{PBC}$ and APBC indeed correspond to two different topological sectors of a Dirac spin liquid, whose energy splitting vanishes algebraically with the system size. However, because of the semimetallic parton band structure, the response is quite different: Any twist of the parton boundary conditions will shift the energy by $v_{F} L_{x} / L_{y}^{2}$, where $v_{F}$ is the spinon velocity, because of the energy of the filled parton bands below the Dirac point. In particular, a boundary condition in which the allowed $k$ modes avoid the Dirac point will be the lowest in energy since this 
effectively opens up a gap of order $v_{F} / L_{y}$. Thus, when a Dirac spin liquid is placed on a narrow cylinder, the gauge field will generically adjust to open up a gap. Therefore, a numerical observation of a nonvanishing gap on a single long cylinder generally does not rule out the possibility of a gapless DSL.

Here, flux threading can be used to find fingerprints of a DSL on a cylinder. As before, adiabatically threading spin flux $\theta$ through the cylinder twists the up or down parton boundary conditions by $\pm \theta / 2$, as shown in Fig. 2(a). This twist is in addition to the (shared) emergent flux $\phi$. For symmetry reasons, the internal gauge flux will either be $\phi=0$ or $\pi$. As we thread spin flux, the energy spectrum will again generically look like Fig. 2(b), but in contrast to a gapped SL, the splitting is only algebraically small. Beyond the crossing, it will become difficult to adiabatically track the state because of the large splitting and small gap (since the allowed $k$ modes become close to the Dirac point); at some point, the $\pi$-emergent flux will enter to partially cancel the spin flux, at which point there will be a discontinuous jump in the ground-state energy of the DMRG simulation [60].

The expected behavior of the gap depends on the geometry, which we consider in more detail at the mean-field level in order to interpret our numerical results. At spin flux $\theta$, the momenta of the partons $f_{\uparrow / \downarrow}$ on a YC2n-2m cylinder are restricted to

$$
n k_{1}+m k_{2}=\phi \pm \theta / 2 \bmod 2 \pi,
$$

where $\phi=0, \pi$ is the emergent gauge flux. The \pm correspond to spinons $f_{\uparrow}, f_{\downarrow}$, respectively. There are two classes of cylinders that behave very differently with $\theta$ :

(i) Type I cylinder: $\mathrm{YC} 2 \mathrm{n}-4 \mathrm{k}$. For $\mathrm{YC} 2 \mathrm{n}-4 \mathrm{k}$ cylinders, when $\theta=0$ the flux $\phi=\pi$ avoids both Dirac points, while both are present for $\phi=0$. In this case, we would (naively) expect the gap to decrease like $(2 \pi-\theta) / L_{x}$ until adiabaticity is lost after $\theta>\pi$ and the emergent $\pi$ flux tunnels in. Hence, we cannot force the system to go gapless.

(ii) Type II cylinder: YC2n-(4k+2). In contrast, the YC2n- $(4 \mathrm{k}+2)$ cylinder becomes gapless at $\theta=\pi$. Here, for $\phi=0$, the $\uparrow Q$ and $\downarrow Q^{\prime}$ components are gapless, while for $\phi=\pi$, the $\downarrow Q$ and $\uparrow Q^{\prime}$ are gapless. All other values of spin flux have a gap that should decrease as $|\pi-\theta| / L_{x}$. Thus, at $\theta=\pi$, two of the four Dirac fermions are present regardless of the emergent flux, and hence, we can force the system to go gapless.

\section{NUMERICAL RESULTS}

In the following, we discuss the numerical results for the KAH obtained using DMRG simulations on infinite cylinders (iDMRG) [47]. Besides the nearest-neighbor Heisenberg interactions, we also include a small secondneighbor interaction for some simulations,

$$
H=J_{1} \sum_{\langle i j\rangle} \vec{S}_{i} \cdot \vec{S}_{j}+J_{2} \sum_{\langle\langle i j\rangle\rangle} \vec{S}_{i} \cdot \vec{S}_{j}
$$

We study the behavior of the spin gap and transfer matrix spectrum (an analog of the excitation spectrum) as we adiabatically twist the boundary conditions (spin-flux $\theta$ ). We implement the adiabatic twist by using the previous DMRG wave function as the initial step for the next $\theta$ value [60]. The ground-state energy during the insertion is provided in Appendix B 2a. Note that while DMRG generally finds the absolute ground state, when passing adiabatically through a level crossing, there may be a small regime in which the DMRG follows the higher, metastable level until "tunneling" into the lower state, a phenomenon we encounter below. We find that the behavior of all the geometries can be grouped into the two types discussed in Sec. III C. For the type I cylinders (YC8-0 and YC8-4), the adiabaticity fails at $\theta \approx 4 \pi / 3$, and the simulation collapses to the other topological sector with lower energy [60]; note for $\pi<\theta \lesssim 4 \pi / 3$, we are tracking the higher, metastable state, not the absolute ground state. For type II (YC6-2, YC8-2, YC10-2, YC12-2, and YC8-6), the adiabaticity fails around $\theta \approx \pi$, at which point we find an instability of the kagome spin liquid towards an ordered state. For a DSL, this instability corresponds to a spontaneous generation of mass $i \bar{\Psi} \sigma^{3} \mu^{3} \Psi$ (see Appendix C for more details). This instability can itself serve as an indication for a Dirac spin liquid, as a gapped spin liquid would not be prone to it.

\section{A. Spin gap}

The spin (triplet) gap $\Delta_{S=1}$ is obtained by creating an $S^{z}=1$ excitation in the bulk of the cylinder and then calculating the energy difference to the $S^{z}=0$ sector [36,37] (see Appendix A for details). We stress here that the spin gap is different from the (extensive) energy splitting between the different topological sectors.

Figure 3(a) provides raw data for the spin gap as a function of the twist angle $\theta$ for DMRG bond dimension $m=4000$. Generally, the spin gap decreases with increasing bond dimension, as seen in Fig. 3(b), and thus the data shown provide only an upper bound for the spin gap. When $\theta=0$, the spin gap is rather large: For example, YC8-0 and YC8-4 have a spin gap of $\Delta_{S=1} \approx 0.15 \mathrm{~J}$, completely consistent with previous DMRG simulations [8,11]. Strikingly, the spin gap shows a significant decrease when $\theta$ is increased, and right before the failure of adiabaticity, the spin gap drops to a very small value $\Delta_{S=1} \approx 0.02 \mathrm{~J}-0.04 \mathrm{~J}$. The approximately linear decrease of the gap for larger twist angles $\theta$ is suggestive of a DSL gapless spin liquid. As discussed in the previous section, the spin gap for a gapped spin liquid should have an exponentially small $\left[\mathcal{O}\left(e^{-L_{y} / \xi}\right)\right]$ dependence on the twist angle $\theta$, though of course $\xi$ can be large.

We emphasize that, based on our data alone, we cannot conclude whether the gap will vanish in the thermodynamic limit. For large enough $L_{y}$, the spin gap of a DSL at $\theta=0$ should decrease with the circumference size $L_{y}$ as 

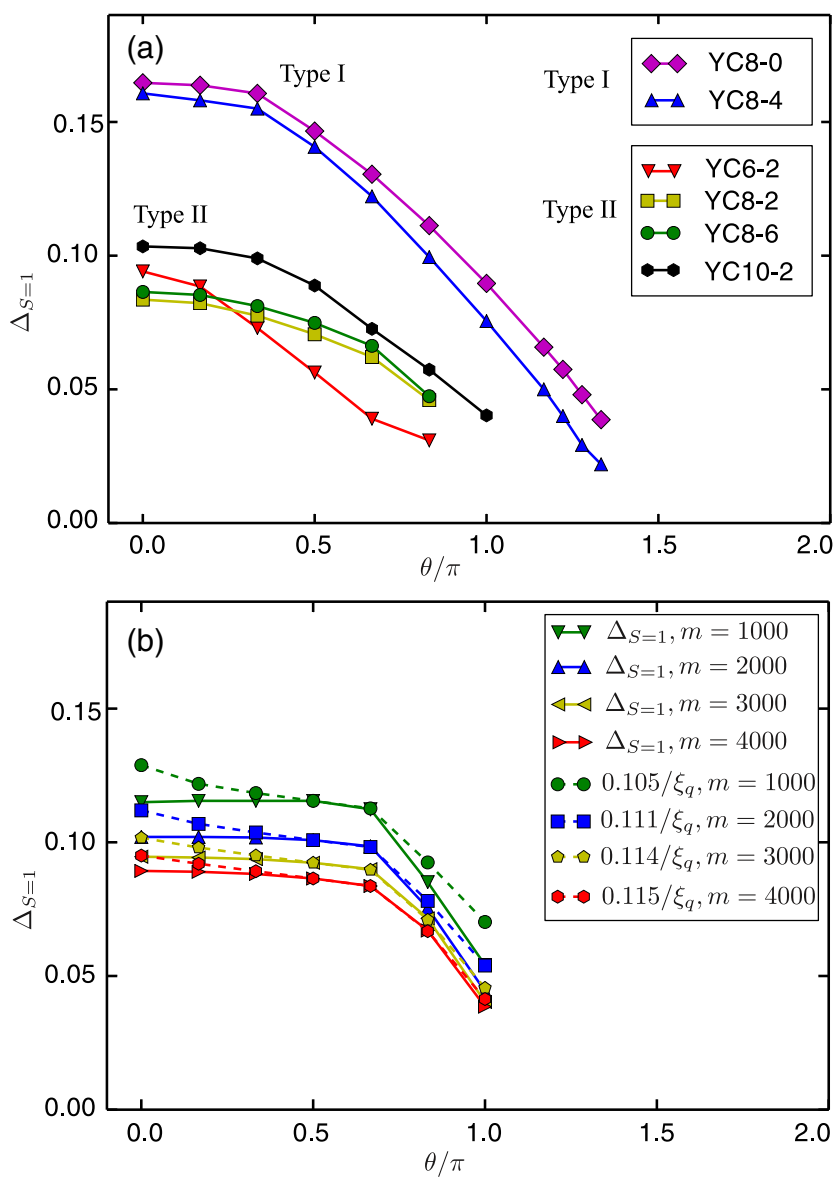

FIG. 3. (a) Upper bound on the spin gap $\Delta_{S=1}$ under the twist boundary condition $\theta$ for $J_{2}=0$; the estimate is obtained using DMRG bond dimension $m=4000$ and using the lowest-energy topological sector. Data end at the failure of adiabaticity. The qualitative behavior depends on the type of cylinder (I or II), which in a DSL would be gapless at $\theta=2 \pi, \pi$, respectively. (b) Dependence of the spin gap $\Delta_{S=1}$ (solid line) and $S^{z}=1$ correlation length $\xi$ (dashed line) on the spin flux $\theta$ and DMRG bond dimension $m$. Data are taken with $J_{2}=0.05$ on the type II YC8-2 cylinder. Generally, the estimated gap decreases with the bond dimension $m$; the larger the system size is, the more likely we are to overestimate the spin gap.

$\Delta_{S=1} \sim v_{F} / L_{y}$. This behavior has not been observed in previous studies $[8,11]$ nor in our current simulations. On the one hand, this could be an artifact of the finite bond dimension $m$ of DMRG simulations; finite $m$ tends to overestimate the spin gap [Fig. 3(b)], necessitating a careful extrapolation in $1 / m^{\alpha}$, where $\alpha$ is an unknown power. Since $m$ must increase exponentially with circumference to achieve the same accuracy, it becomes very challenging to accurately extract the spin gap for large circumferences. It is also the reason why the larger system size appears to have a larger gap in Fig. 3. Indeed, at comparable numerical accuracy, the larger system size has a smaller spin gap (see Appendix B 2e, Fig. 16). Alternatively, for small circumferences, it is unclear whether the gap will follow the naive mean-field expectation $\Delta_{S=1} \sim v_{F} / L_{y}$; a possible reason is discussed in Sec. IV B 1.

\section{B. Transfer matrix spectrum}

We examine the transfer matrix spectrum on the infinite cylinder (i.e., the correlation-length spectrum) and compare the KAH with a free-fermion $\pi$-flux model. In the ansatz wave function of the DMRG (matrix product states), the correlation functions of charge- $q$ operators [e.g., the spinspin correlations $\left.C_{S=1}(r)=\left\langle S_{0}^{+} S_{r}^{-}\right\rangle_{\text {con }}\right]$ can be expanded as a sum of exponentials,

$$
C_{q}(r)=\sum_{j=1}^{m_{q}} \alpha_{q, j} \lambda_{q, j}^{r}
$$

Here, $r$ is the distance along the long direction of the cylinder, $q$ is a quantum number, $m_{q}$ is a bond dimension, and $\lambda_{q, j}$ are eigenvalues of the DMRG transfer matrix [61]. Using the quantum numbers $q$, we can distinguish, for example, a triplet excitation from a singlet excitation (or, more precisely, an $S^{z}=1$ excitation from a $S^{z}=0$ excitation). The eigenvalues $\lambda_{q, j}=e^{i k_{q, j}-\xi_{q, j}^{-1}}$ have a real part, corresponding to a correlation length $\xi_{q, j}$, and an imaginary part, corresponding to a momentum $k_{q, j}$. The largest, $\xi_{q}=\max \left(\xi_{q, j}\right)$, bounds the correlation length of all charge- $q$ operators.

A recent work by Zauner et al. [48] pointed out a relation between the energy spectrum of the physical excitations and the spectrum of the transfer matrix. A more familiar statement is that the largest correlation lengths $\xi_{q}$ set an upper bound for the lowest excitation gaps $\Delta_{q}$ (up to a factor), and for a Lorentz-invariant system, it holds that $\Delta_{q} \propto 1 / \xi_{q}$. The corresponding $k_{q}$ gives the momentum of the excitation along the length of the cylinder. These relations actually hold nicely for the KAH at different twist angles, as demonstrated in Fig. 3(b).

Figures 4(a) and 4(b) show the $S^{z}=1$ transfer matrix spectrum of the KAH and the $\pi$-flux free-fermion model Eq. (3) as functions of the twist angle $\theta$. We consider the type II YC8-2 geometry and include a small $J_{2}$ to stabilize the adiabatic twist up to $\theta=\pi$ (Appendix B 2e, B 2d shows results for other geometries). The three different colors label three "sectors" distinguished by their momenta; we observe that the momenta $k_{S=1, j}$ cluster into three distinct groups, and we plot the largest several $\xi_{S=1, j}$ from each momenta group. The momentum can be resolved into its lattice components $k_{1}, k_{2}$, providing an alternative way to plot the data. In Figs. 4(c)-4(f), for each twist angle $\theta$, we choose only the largest correlation length $\xi$ in each of the three different sectors (colors) and plot them in momentum space. The Dirac point in momentum space corresponds to the data at the twist angle $\theta=\pi$.

\section{Interpretation as DSL}

The KAH and free-fermion spectra are remarkably similar. The excitation spectrum can be understood based 

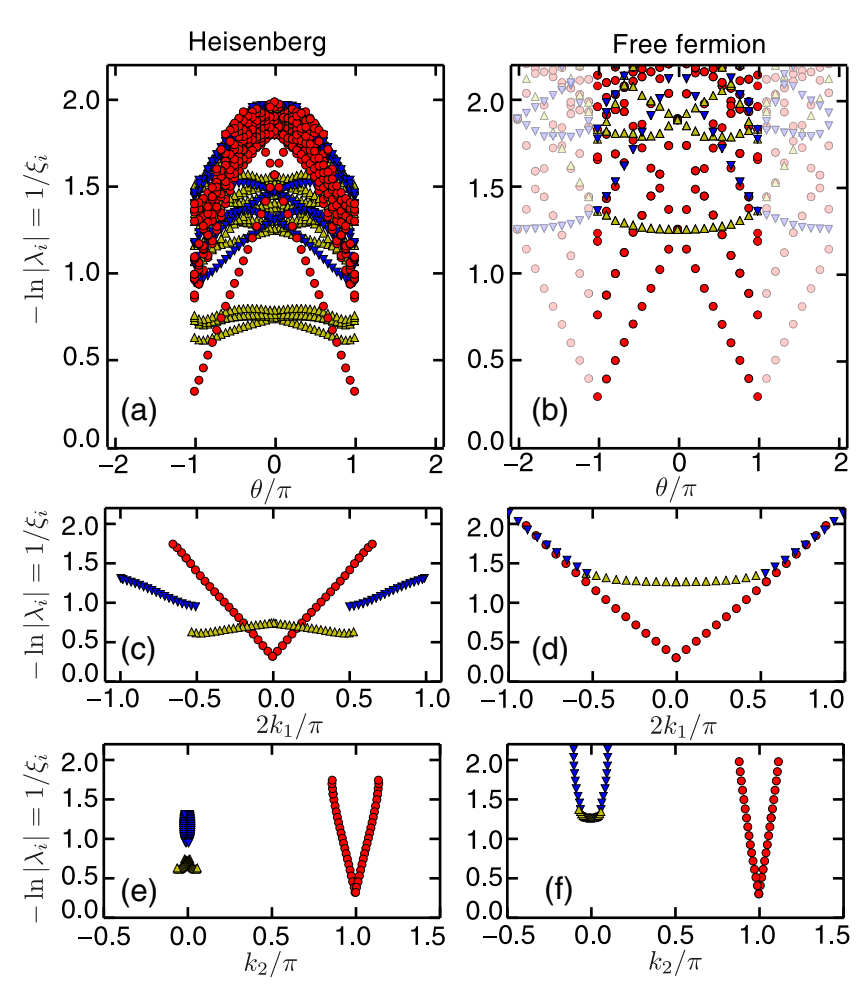

(g)

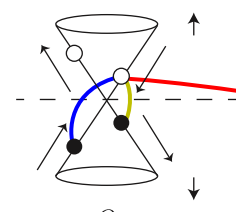

$Q$

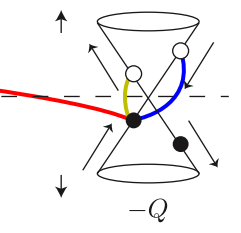

FIG. 4. The transfer matrix spectrum in the $S^{z}=1$ sector (roughly the triplet channel) for the kagome Heisenberg model $(\mathrm{a}, \mathrm{c}, \mathrm{e})$ and free-fermion model $(\mathrm{b}, \mathrm{d}, \mathrm{f})$. The vertical axis is the inverse of correlation length $1 / \xi$, which can be considered as the gap $\Delta$ of the excitations, up to a prefactor $\Delta=v_{s} / \xi$. The horizontal axis denotes $(\mathrm{a}, \mathrm{b})$ the twist angle $\theta ;(\mathrm{c}, \mathrm{d})$ momentum $2 k_{1}$; and (e,f) momentum $k_{2}$. In panels (c)-(f), we plot only the lowest level of each excitation type, $\mathrm{cf}$. panel (g). The cylinder we show here is the YC8-2 cylinder for both cases, and the truncation error of DMRG is around $2 \times 10^{-6}$, which corresponds to bond dimension $m=6000$ for the kagome Heisenberg model and $m=250$ for the free-fermion model. For the kagome Heisenberg model, we also include a small $J_{2}=0.05$. The finite "gap" at the Dirac point appears to be due to the truncation error of DMRG simulations (see Fig. 15 in Appendix B 2e). (g) Three different types of particle-hole excitations. The arrows represent the direction of the movement of the discretized momentum under the twist boundary conditions.

on the free-fermion $\pi$-flux model. Note that $S^{z}=1$ excitations arise from particle-hole excitations near the Dirac points; a momentum $p-q$ spin flip takes the form $S^{+}(p-q)=f_{\uparrow}^{\dagger}(p) f_{\downarrow}(q)$. The $\pi$-flux state has two Dirac points at $Q=(\pi / 2, \pi / 2)$ and $Q^{\prime}=(-\pi / 2,-\pi / 2)$. We group the particle-hole excitations into intravalley forward (blue), intravalley backward (yellow), and intervalley forward (red), as illustrated in Fig. 4(g) (intervalley backward scattering is higher in energy). The Dirac points are avoided on the $\theta=0$ YC8-2 cylinder, as shown in Fig. 1(d), but as $\theta$ increases, the allowed momenta shift and eventually pass through the Dirac point; the $f_{\uparrow}$ and $f_{\downarrow}$ feel opposite flux and hence move oppositely. As can be seen in figure, this shift affects the three modes in a qualitatively different fashion. The dispersion of the red mode follows a Dirac behavior and becomes gapless at the twist angle $\theta= \pm \pi$. In terms of momenta, the gapless point occurs at $\left(2 k_{1}, k_{2}\right)=(0, \pi)$ as expected from the displacement between $Q$ and $Q^{\prime}$. The yellow mode has a constant energy under the twist angle $\theta$. The blue mode has a similar response as the red mode, but it remains gapped when the system hits the Dirac points $(\theta=\pi)$.

The spectrum of the KAH and the $\pi$-flux free-fermion model show surprisingly good agreement: (i) The red mode has a linear sharp Dirac cone structure, (ii) the yellow mode is almost flat, and (iii) the modes occur with the predicted momenta. The qualitative difference between the two models is that the yellow and blue modes in the KAH are lower compared with the free-fermion model. Even though the DSL theory should have an emergent $S U(4)$ symmetry in $2 \mathrm{D}$, in the quasi-1D geometry, the intravalley interactions may be stronger.

The existence of the renormalized flat yellow band also explains the kink in the $\theta$ dependence of the triplet gap $\Delta_{S=1}$ : For small $\theta$, it drops below the linear red band; the two bands then cross. This implies that gaps obtained in previous DMRG studies, which all worked at $\theta=0$, were probing the yellow intravalley excitation. Since the yellow band is subject to strong interaction effects, this may relate to the nonobservation of $v_{F} / L_{y}$ gap scaling on accessible cylinders.

We want to remark that within our DMRG simulations, the correlation spectrum of the KAH still has a finite "gap" even at the Dirac point. This is a necessary consequence of DMRG since the finite bond dimension $m$ induces a finite correlation length. We find the $\xi$ increase with $m$ (see Appendix B 2e, Fig. 15), as expected for a DMRG simulation of a critical system. In fact, a similar behavior is also found in the free-fermion model. The correlation length estimated from DMRG (with $m=250$ in Fig. 4) is finite even for the gapless free-fermion model with an infinite correlation length. For a very large bond dimension ( $m=3000$; see Appendix B 1, Fig. 8), the correlation length becomes very large ( $\xi \sim 1000$ sites), supporting the fact that the finite correlation length at the Dirac point (in Fig. 4) is purely an artifact of small bond dimension.

The spectrum we find here is qualitatively different from the transfer matrix of the gapped $Z_{2}$ spin liquid reported in Ref. [62]. For additional comparison, we also include the transfer matrix for the mean-field ansatz of the chiral spin liquid, i.e., a gapped Chern band that descends from the $\pi$-flux ansatz (Appendix B 1, Fig. 8), as well as a magnetic ordered state (Appendix B 2f, Fig. 17). 

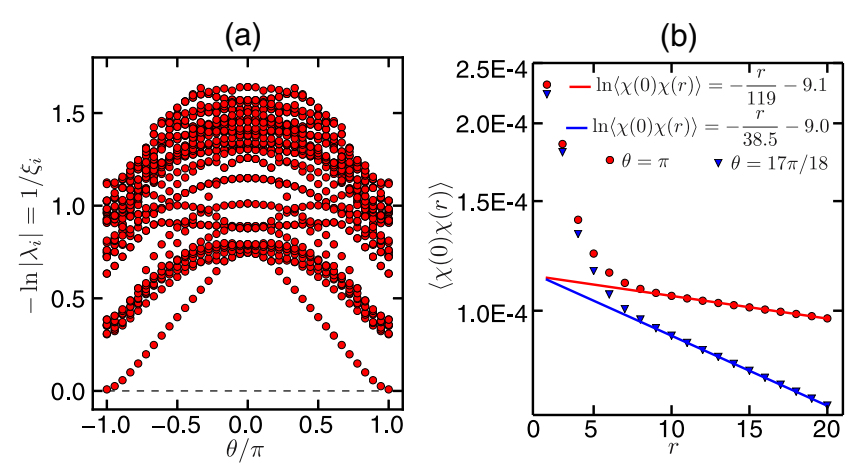

FIG. 5. (a) The transfer matrix spectrum of the kagome Heisenberg model in the $S^{z}=0$ sector versus the twist angle $\theta$. Here, we have the YC8-2 cylinder, $J_{2}=0.05$, and bond dimension $m=6000$. (b) Correlation function of the scalar chirality $\langle\chi(0) \chi(r)\rangle$ at $\theta=\pi$ and $17 \pi / 18$. The correlation lengths from fitting $\langle\chi(0) \chi(r)\rangle$ are $\xi=119(\theta=\pi)$ and $\xi=38.5$ $(\theta=17 \pi / 18)$, which are virtually identical to the correlation lengths obtained from the transfer matrix $\xi=121$ and $\xi=39$.

\section{C. $S^{z}=0$ spectrum and scalar chirality}

We now turn to the $S^{z}=0$ transfer matrix spectrum shown in Fig. 5(a), which includes singlet excitations for which the interactions have a more drastic effect. Note that because our numerics do not explicitly preserve $S O(3)$ symmetry, the $S^{z}=0$ spectrum contains both $S O(3)$ singlets and elements of $S O(3)$ multiplets. The correlation length in the $S^{z}=0$ sector shows a critical feature: At the twist angle $\theta=\pi$, the $S^{z}=0$ correlation length is $\xi \sim 100$ unit cells, which (holding fixed the bond dimension $m=6000$ ) is much larger than the correlation length $\xi \sim 5$ in the $S^{z}=1$ sector. This effect is beyond a meanfield analysis since, for a free fermion, there will be no difference between $S^{z}=0$ and $S^{z}=1$ sectors.

The large correlation length in the $S^{z}=0$ sector arises from long-range correlations of the scalar chirality $\chi=\vec{S}_{i}\left(\vec{S}_{j} \times \vec{S}_{k}\right)$. To confirm this, in Fig. 5(b), we show a fit for the correlation function of the scalar chirality, $\langle\chi(0) \chi(r)\rangle \sim e^{-r / \xi}$. Indeed, the correlation length $\xi$ is almost identical to the largest correlation length obtained from the transfer matrix in the $S^{z}=0$ sector. The large correlation length of the scalar chirality is somewhat surprising given our knowledge of $N_{f}=4 \mathrm{QED}_{3}$ [U(1) DSL]. The scalar chirality corresponds to the $S U(4)$ singlet $\bar{\psi} \psi$, which was suggested to have higher scaling dimension than the $S U(4)$ adjoint fermion bilinears [63]. Naively, an operator with a lower scaling dimension in a critical theory would give a larger correlation length in DMRG calculations with a finite bond dimension, in an apparent contradiction with our result. However, our result does not necessarily indicate that the scalar chirality has the lowest scaling dimension in the critical theory since the magnitude of the correlation length is not directly equivalent to the scaling dimension of an operator. Another possible explanation is that the cylinder geometry drastically changes the scaling analysis since (if it was stable)
$\mathrm{QED}_{3}$ dimensionally reduces to $N_{f}-1$ coupled TomonagaLuttinger liquids [64], for which the scaling analysis may differ substantially from the 2D limit. In addition, we note that the $S U(4)$-invariant mass $\bar{\psi} \psi$ generates the CSL (it gives the bands a net Chern number), and we know that the KAH is proximate to the CSL $[19,37,38,65]$. It was also suggested that the scalar chirality contains a monopole operator, which might have a lower scaling dimension than the fermion bilinears [6].

\section{CONCLUSION AND DISCUSSION}

We used large-scale DMRG simulations to study the quantum spin-liquid phase in the $S=1 / 2$ kagome antiferromagnetic Heisenberg model. DMRG studies of the KAH are most natural on a cylinder. We point out that, even if a gapless QSL such as the Dirac spin liquid is realized in the two-dimensional bulk limit, the system generally acquires a nonvanishing gap on the cylinder. The predicted size of this gap depends sensitively on the geometry and boundary conditions of the cylinder, complicating the finite-size scaling analysis. Thus, the observation of a gap in the previous DMRG studies of the KAH, which is also reproduced in our study, might not rule out a gapless QSL until the gap can be accurately measured for a sequence of "equivalent" geometries (e.g., YC8, YC12, YC16, ...), which is extremely challenging because of the exponential blowup in DMRG bond dimension.

To better identify the nature of the QSL in the KAH using DMRG, we insert spin flux through the cylinder, changing the boundary condition around the circumference. Using adiabatic flux insertion, we find that the spin gap on the cylinder geometry is much smaller than estimated from previous DMRG simulations. Second, we found that the momentum-dependent excitation spectrum, as estimated from the DMRG transfer matrix, exhibits Dirac cones that agree well with the ones found for a $\pi$-flux free-fermion model [the parton mean-field ansatz of a $U(1)$ Dirac spin liquid]. These findings suggest that the ground state of the KAH is a gapless DSL, instead of a gapped QSL such as the $\mathbb{Z}_{2}$ topological phase. This is more in line with several recent numerical $[5,10,14-17,22,23]$ and analytical [41] results obtained by methods other than DMRG.

Besides the direct numerical evidence summarized above, there is other circumstantial evidence that the KAH is a $U(1)$ Dirac spin liquid. First, we observe an instability of the kagome spin liquid to an ordered state at the twist angle $\theta=\pi$ in the YC4k-2 cylinder. This instability can be explained as the spontaneous mass generation $\left(i \bar{\Psi} \sigma^{3} \mu^{3} \Psi\right)$ of Dirac fermions on a small cylinder. Second, we find that the excitations in the singlet sector are very different from the triplet sector, which may be because of the $U(1)$ gauge field (either the photon or the monopole). Third, early studies found a chiral spin liquid $[19,37,38,65]$ proximate to the kagome spin liquid, and the transition between them is consistent with being continuous [19]. This can be 
understood as a spontaneous mass generation $(i \bar{\Psi} \Psi)$ for the Dirac fermions in a $U(1)$ DSL. In contrast, there is no known theory for such a transition from a gapped $Z_{2}$ spin liquid (with toric-code topological order) or a $Z_{2}$ Dirac spin liquid.

However, we also have to give a word of caution. While we found indications of gapless features in the system sizes that we can access, we cannot draw a definite conclusion for the thermodynamic limit. Diverging correlation lengths require extremely large bond dimensions for proper convergence of the gap, making it impossible to rule out the existence of a small but finite gap with a large correlation length. In addition, fixing $\theta=0$, the spin gap should eventually decrease as $v_{F} / L_{y}$, which has not yet been observed. Within a DSL scenario, this could be a finite-size effect due to observed strong renormalization of the intravalley backward scattering, or it could be a numerical artifact of finite-bond dimension. Finally, the present data do not directly reveal the nature of the gauge field, which could be either $U(1)$ or $\mathbb{Z}_{2}$. It would be very interesting if the details of the entanglement spectrum, as well as an analysis of the other low-energy states, could be used to infer such information. Therefore, our result is certainly not the final answer to the long-standing question on the KAH. Nevertheless, our results strongly suggest that the ground state of the KAH is a DSL, which has many theoretical and experimental implications.

\section{ACKNOWLEDGMENTS}

We thank M. Barkeshli, Y.M Lu, M. Metlitski, A. Vishwanath, and C. Wang for useful discussions. M.Z. thanks D. Huse and S. White for collaboration on related work. Y.C. H. thanks the Institute for the Solid State Physics, University of Tokyo, where this project was initiated, for hospitality. Y. C. H. is supported by a postdoctoral fellowship from the Gordon and Betty Moore Foundation, under the EPiQS initiative, GBMF4306, at Harvard University. This work was supported in part by JSPS Strategic International Networks Program No. R2604 "TopoNet" (Y.C.H. and M. O.), JSPS Grant-in-Aid for Scientific Research (KAKENHI) No. 16K05469 (M. O.), and DFG via SFB 1143. The work of M. Z. was performed in part at the Aspen Center for Physics, which is supported by National Science Foundation Grant No. PHY-1066293. Y. C. H., M. Z., M. O., and F. P. acknowledge the hospitality of the Kavli Institute for Theoretical Physics, which is supported in part by the National Science Foundation under Grant No. PHY-11-25915.

\section{APPENDIX A: NUMERICAL ALGORITHM}

\section{Transfer matrix and its spectrum}

Numerically, we wrap a 2D lattice on a cylinder with one direction compactified with a small number of sites, and the other direction is infinite. We use the MPS to cover the cylinder in the fashion of a snake chain, as graphically
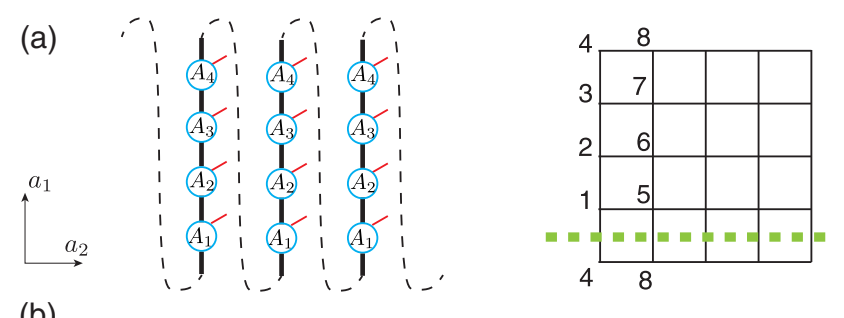

(b)

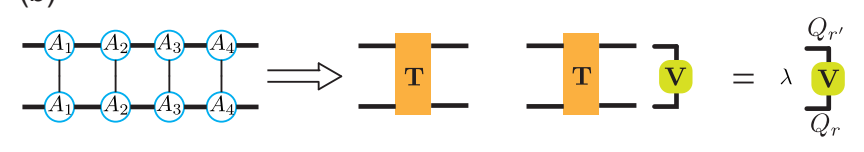

(c)



(d) 4

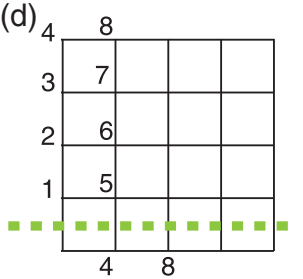

(e)

FIG. 6. The graphical representation of (a) the MPS and (b) the transfer matrix. The quantum number of the eigenvalue is determined by $q=Q_{r}-Q_{r^{\prime}}$. (c) The mixed transfer matrix to calculate the momentum $k_{1}$ of each Schmidt basis. (d) The -1 geometry for the square lattice (it is analogous to the -2 geometry for the kagome lattice). The periodic condition is found by identifying the sites labeled by the same number. (e) The mixed transfer matrix for the fermionic excitation.

shown in Fig. 6(a). Along the compactified direction $\left(a_{1}\right)$, the snake covering does not maintain the translational symmetry explicitly; thus, one needs distinct MPS for each site. On the other hand, the MPS is translation invariant and repeating along the direction $a_{2}$. Then, one can use the MPS (of the smallest repeating unit cell) to define the transfer matrix (TM), as shown in Fig. 6(b). With the TM, one can further calculate its eigenvalues $\lambda_{q, j}=e^{i k_{q, j}-\xi_{q, j}^{-1}}$, which have a real part, corresponding to a correlation length $\xi_{q, j}$, and an imaginary part, corresponding to a momentum $k_{q, j}$. Here, $q$ are the quantum numbers, from which we can distinguish, for example, triplet excitation from singlet excitation.

Because of the snake covering, the MPS does not have translational invariance along the compactified direction $\left(a_{1}\right)$. However, the Hamiltonian still has translational invariance along $a_{1}$; hence, the momentum $k_{1}$ along that direction can still be extracted. The way to calculate $k_{1}$ is similar to calculating a global quantum number $q$, for which one needs to obtain the momentum $k_{1}$ of each Schmidt basis. Technically, we implement the translational operation $T_{1}$ along the direction $a_{1}$ and then obtain a mixed TM-T ${ }^{T_{1}}$, as shown in Fig. 6(c). The dominant eigenvector 
of $\mathbf{T}^{T_{1}}$ will be $\mathbf{V}_{\alpha, \beta}=\delta_{\alpha, \beta} e^{i k_{\alpha}}$, and $k_{\alpha}$ gives the momentum $k_{1}$ of each Schmidt basis.

We remark that for certain special geometries, for instance, the YC2n-2, the momentum along the compactified direction cannot be extracted using the method discussed above because, for those geometries, the momenta $k_{1}$ and $k_{2}$ are intertwined together. A consequence of this is that one cannot define the mixed TM for the translation $T_{1}$. For example, we can consider the -1 geometry for the square lattice, Fig. 6(d) (it is the analogy of the -2 geometry for the kagome lattice). One can see that under the $T_{1}$ translation, the sites on one column do not go back to the same column; instead, some of the sites will go to the neighboring column. This is different from the normal geometry [see the left panel of Fig. 6(a)], for which $T_{1}$ translation maps the sites on one column back to itself; hence, the mixed TM $-\mathbf{T}^{T_{1}}$ can be well defined. A benefit of the -2 geometry, however, is that the snake-fashion MPS is actually translational invariant under two sites, no matter how large the circumference of the cylinder is. Then, one can actually use a MPS with two-site structure (for the kagome lattice, it is six sites) to do the iDMRG simulation. With the momentum $k$ from the TM's eigenvalue, one can then obtain the momentum

$$
2 k_{1}=k+2 \theta / L_{y}, \quad k_{2}=k L_{y} / 2 .
$$

Here, $L_{y}$ is the width of the cylinder, and $\theta$ is the twist boundary condition. Therefore, for the -2 geometry, one can still get $k_{1}$ and $k_{2}$, but $k_{1}$ has a $\pi$ ambiguity.

Before closing this section, it is worth making a few remarks on fermionic systems. Usually, to simulate a fermionic system, we would first do a Jordan-Wigner transformation to obtain the corresponding bosonic model; we would then simulate the bosonic model directly. Therefore, to calculate the spectrum of the fermionic (e.g., single-particle) excitations, one should consider the mixed $\mathbf{T M}-\mathbf{T}^{f}$, with a Jordan-Wigner string inserted [see Fig. 6(e)].

\section{Algorithm of calculating the spin gap}

We use an algorithm that combines iDMRG and finite DMRG to calculate the spin gap. First, we use iDMRG to obtain a converged wave function of an infinite cylinder; then, we cut the infinite cylinder into two halves and insert $3 \times$ $L_{y} \times L_{y}$ sites into the system (as shown in the Fig. 7). The left $(L)$ and right $(R)$ semi-infinite cylinder can be considered the environment (boundary conditions), and we calculate the energy of the ground state $E_{0}\left(S_{z}=0\right)$ and the spin-1 sector $E_{0}\left(S_{z}=1\right)$ within the inserted $3 \times L_{y} \times L_{y}$ cylinder. Finally, we obtain the spin gap $\Delta_{S=1}=E_{0}\left(S_{z}=1\right)-E_{0}\left(S_{z}=0\right)$.

This algorithm is similar to the one used in finite DMRG, where one obtains the spin (triplet) and singlet gap by sweeping in the middle of a finite cylinder to minimize the boundary effect. The only difference is that the boundary environment we use comes from the iDMRG simulation, while finite DMRG uses the environment from finite cylinder simulation.

\section{APPENDIX B: ADDITIONAL NUMERICAL DATA}

\section{Benchmark for free fermions}

We begin with the transfer matrix spectrum of the freefermion model, the $\pi$-flux state on the kagome lattice [Eq. (3)]. Here, we show the data with a larger bond dimension $m=3000$ (Fig. 8); meanwhile, we also compare the case where the ground state is a Chern insulator. It is clear that, compared with the small bond dimension data $[m=250$ in Figs. 4(b), 4(d), and 4(f)], the spectrum of free Dirac fermions [Figs. 8(a), 8(c), and 8(e)] is almost critical at the Dirac point. This supports the fact that the finite correlation length of the kagome Heisenberg model at the Dirac point is simply an artifact of finite bond dimension in the DMRG

(a)

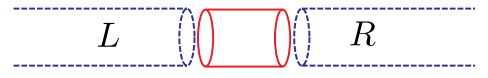

(b)

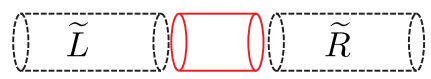

FIG. 7. (a) iDMRG-finite DMRG combined algorithm. (b) Finite DMRG algorithm.
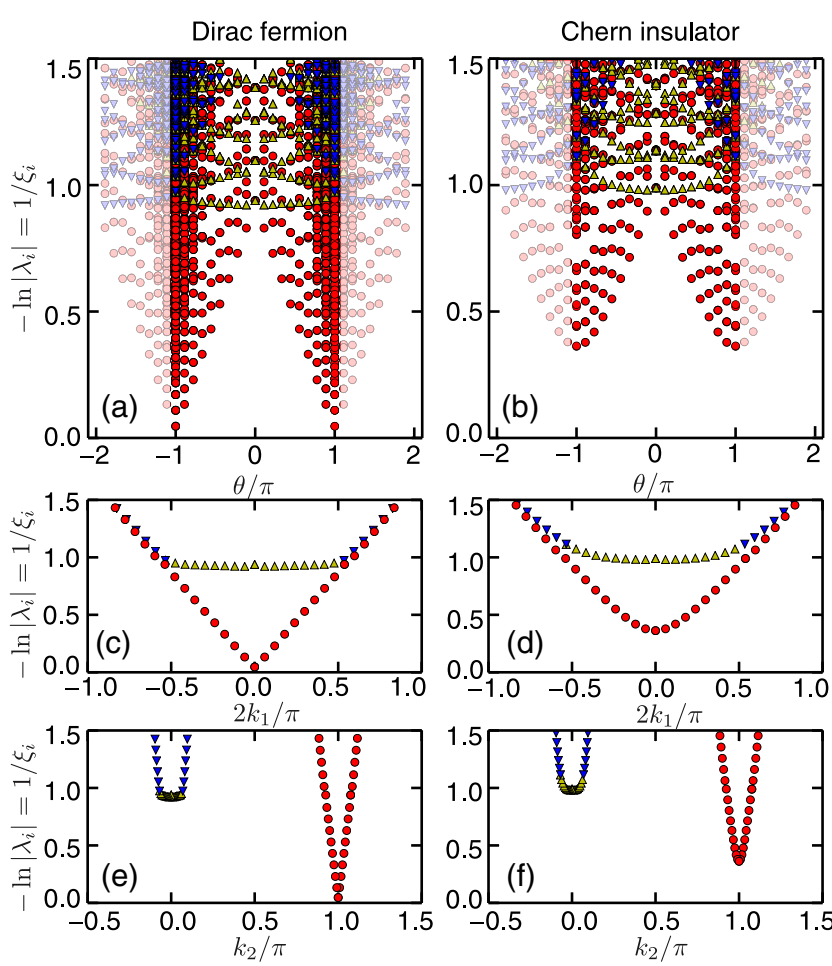

FIG. 8. Transfer matrix spectrum for free fermions [Eq. (3)] on the YC8-2 cylinder with bond dimension $m=3000$. Here, we compare the free Dirac fermion model and a Chern insulator: the inverse of correlation lengths $1 / \xi$ versus $(a, b)$ twist angle, (c,d) momentum $2 k_{1}$, and (e,f) momentum $k_{2}$. The Chern insulator we show here is obtained by assigning $\pi / 25$ flux in the up and down triangles (of the kagome), and $23 \pi / 25$ flux in the hexagon. 
simulations. On the other hand, we also calculate the case of a Chern insulator, which clearly shows a parabola shape in the spectrum [Figs. 8(b), 8(d), and 8(f)].

\section{Kagome Heisenberg model a. Spin stiffness}

Figure 9 shows the response of the ground-state energy and entanglement entropy under the twist boundary conditions before the failure of adiabaticity. Practically, the adiabaticity can be checked by looking at the wavefunction overlap $(\approx 0.99)$ between two adjacent twist angles. During the (adiabatic) twist process, the system remains in a spin-liquid phase that preserves all the lattice symmetries. Both the energy and entanglement entropy increase under the twist; the increase in entanglement entropy is very significant and may be underestimated by finite $m$. This behavior is consistent with the DSL. It is worth noting that, for $\mathrm{YC} 8-0$, the twist trajectory is not symmetric about $\theta=\pi$, which is a clear signature of fractionalization; the state at $\theta=\pi$ is not time-reversal invariant either (also true for YC8-2).

\section{b. Transfer matrix}

We provide more data for the transfer matrix spectrum of the KSL. We show that by changing the system sizes and parameter point ( $J_{2}$ interaction), the feature of a Dirac cone structure always exists. For example, the $S^{z}=1$ excitation shows a clear Dirac cone structure. As before, the $S^{z}=0$ excitation is always much lower than the $S^{z}=1$ excitation.

As we described in the main text, the behavior of the system can be grouped into two types, YC2n- $(4 \mathrm{k}+2)$ cylinder and $\mathrm{YC} 2 \mathrm{n}-4 \mathrm{k}$ cylinder. In the following, we look at the two different classes separately.

\section{c. YC2n- $(4 k+2)$ cylinder}

Let us first focus on the YC2n- $(4 \mathrm{k}+2)$ cylinder. As we showed in the main text, this class shows a clear Dirac cone structure in the $S^{z}=1$ sector.

We plot the $S^{z}=1$ transfer matrix spectrum of $J_{2}=$ 0.05 (Fig. 10), $J_{2}=0.1$ (Fig. 11), and $J_{2}=0$ (Fig. 12) for

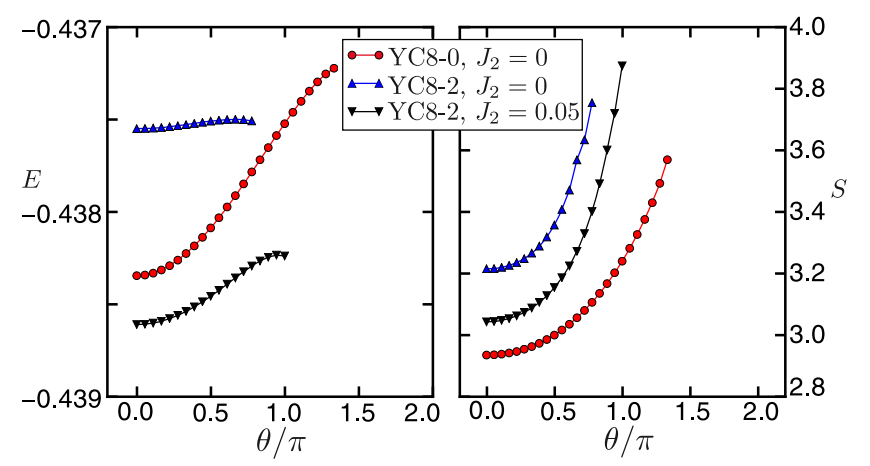

FIG. 9. The energy and entanglement entropy of the KSL under flux insertion. Here, the bond dimension is $m=6000$.
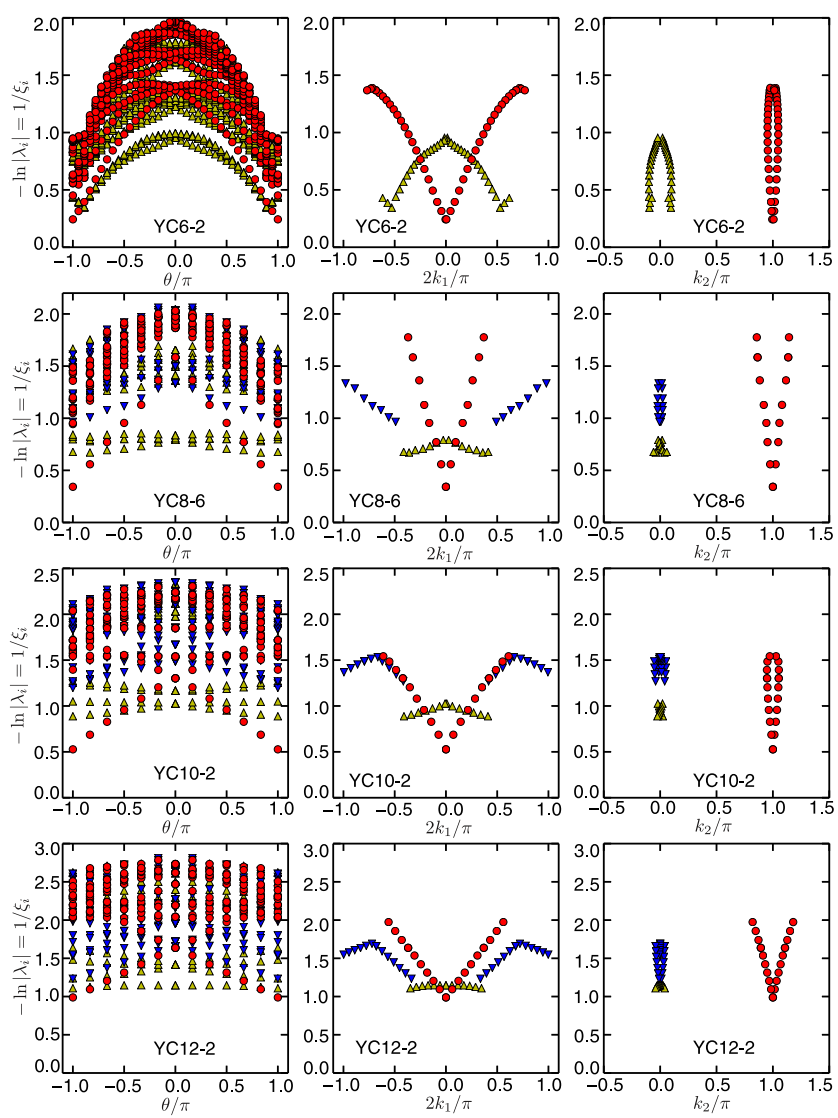

FIG. 10. The $S^{z}=1$ transfer matrix spectrum of $J_{2}=0.05$ for the YC6-2, YC8-6, YC10-2, and YC12-2 cylinders. Here, the bond dimension is $m=6000$.

the YC6-2, YC8-2, YC10-2, YC12-2, and YC8-6 cylinders. Clearly, the Dirac cone is independent of the $J_{2}$ interaction or system sizes. For $J_{2}=0$ in Fig. 12, we find that it is more difficult to maintain the adiabaticity of the twist around $\theta=\pi$. Specifically, for the YC6-2 and YC8-2 cylinders, we can only adiabatically twist the system to $\theta=5 \pi / 6$; for $\theta=\pi$, we always end up with an ordered state with a sudden jump. This is the reason why there are points missing at the Dirac cones in Fig. 12. As we argue in the following section, once a Dirac spin liquid is put on a small cylinder, it may have an instability towards spontaneously generating a mass gap. Such a finite-size effect is absent in the pure $2+1 \mathrm{D}$ limit. This is consistent with our observation that, for a larger system size (i.e., YC10-2 and $\mathrm{YC} 12-2)$, the adiabatic twist can be maintained even for $J_{2}=0$.

We note that in the spectrum, there is a set of four low-lying yellow bands. In the left two panels, where we convert the twist into momentum dependence, for clarity we have only drawn the lowest level in each sector. Therefore, the "peak" in some plot (e.g., YC8-2, $J_{2}=0$ ) is actually a crossing between the four low-lying bands. The dispersion of the yellow mode comes from nonuniversal UV physics such as the quartic interaction of Dirac fermions. 

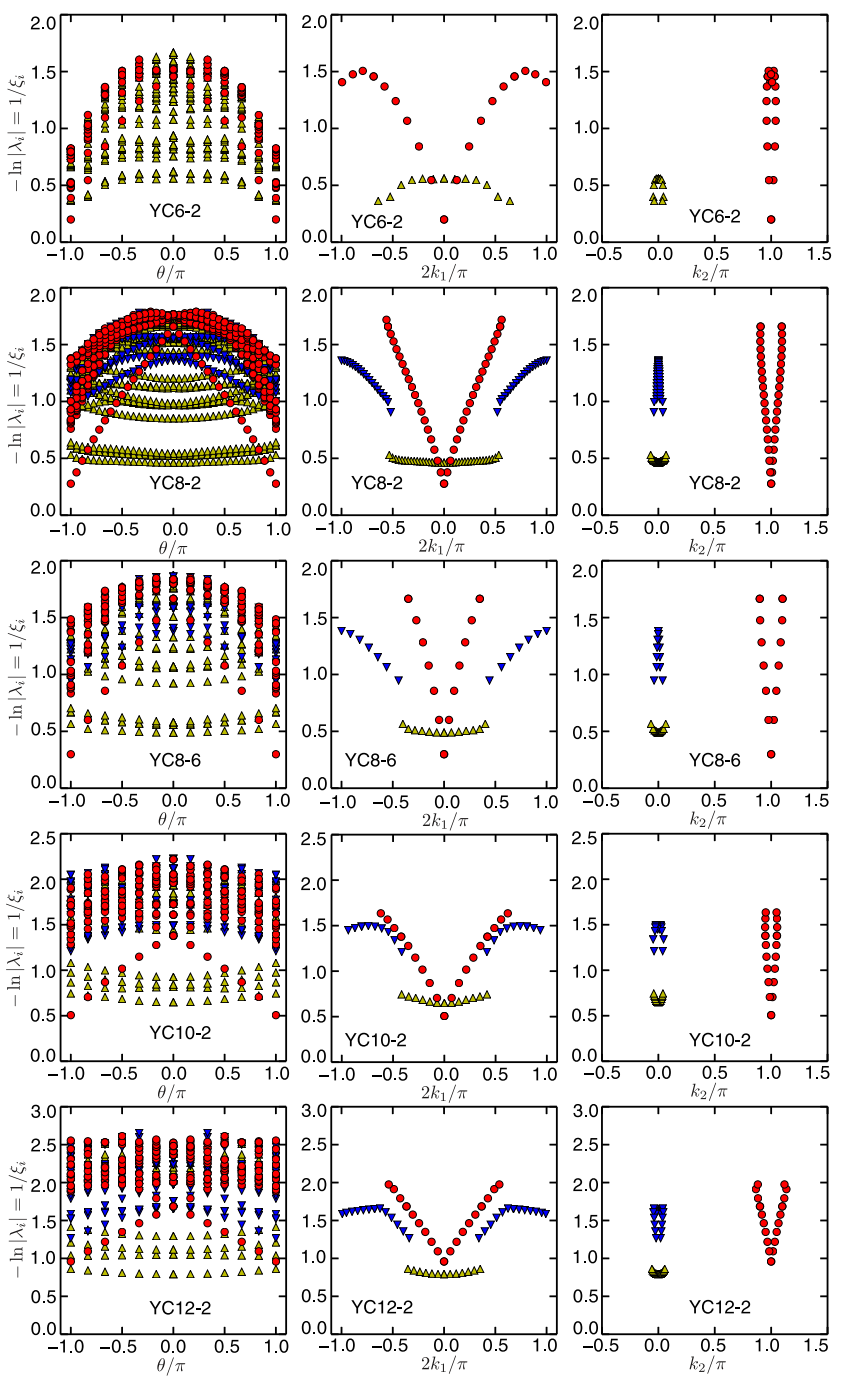

FIG. 11. The $S^{z}=1$ transfer matrix spectrum of $J_{2}=0.1$ for the YC6-2, YC8-2, YC8-6, YC10-2, and YC12-2 cylinders. Here, the bond dimension is $m=6000$.

Besides the $S^{z}=1$ transfer matrix spectrum, it is also interesting to look at the $S^{z}=0$ transfer matrix spectrum, Fig. 13. As we discussed in the main text, those $S^{z}=0$ excitations are more critical than the $S^{z}=1$ excitations. For a large system size, the singlet excitation is not gapless at the Dirac point $\theta=\pi$. We think this is again the artifact of finite bond dimension, as one can see that the singlet excitation keeps decreasing as the bond dimension $m$ increases, as shown in Fig. 13(b).

\section{d. YC2n-4k cylinder}

Finally, let us look at the $\mathrm{YC} 2 \mathrm{n}-4 \mathrm{k}$ cylinder. As we discussed in the main text, this class of cylinder behaves very differently for $\mathrm{YC} 2 \mathrm{n}-(4 \mathrm{k}+2)$, which is discussed above. For the $\pi$-flux Dirac spin liquid, the YC2n- $(4 \mathrm{k}+2)$ cylinder will hit the gapless Dirac point at $\theta=\pi$, while the YC2n-4k cylinder will hit the gapless Dirac point at


FIG. 12. The $S^{z}=1$ transfer matrix spectrum of $J_{2}=0$ for the YC6-2, YC8-2, YC8-6, YC10-2, and YC12-2 cylinders. Here, the bond dimension is $m=6000$.

$\theta=2 \pi$. Our simulation on the $\mathrm{YC} 2 \mathrm{n}-4 \mathrm{k}$ cylinder is also consistent with this scenario; namely, the adiabaticity of the twist can be maintained after $\theta=\pi$ until $\theta \approx 4 \pi / 3$, after which the system collapses to the other topological sector.

When the $\pi$-flux DSL hits the Dirac points at $\theta=2 \pi$ on the YC2n-4k cylinder, the four Dirac fermions will be simultaneously gapless. Those four gapless Dirac fermions could then form different gapless fermion bilinears giving rise to gapless triplet (singlet) excitations. This is again sharply distinct from the $\mathrm{YC} 2 \mathrm{n}-(4 \mathrm{k}+2)$ cylinder, where only two Dirac fermions are gapless when the system hits the Dirac points (at $\theta=\pi$ ). Therefore, for the $\pi$-flux DSL, we expect that the gapless triplet excitation at all three $M$ points will be gapless on the $\mathrm{YC} 2 \mathrm{n}-4 \mathrm{k}$ cylinder. However, we are working in a small cylinder, such that some lattice symmetry (e.g., $C_{3}$ ) is explicitly broken. 




FIG. 13. (a) The singlet excitation spectrum for the (a)-i YC102 and (a)-ii YC12-2 cylinders; here, $J_{2}=0.05$, and bond dimension $m=6000$. (b) The dependence on the bond dimension $m$ of the lowest singlet excitation spectrum for the (b)-i YC10-2 and (b)-ii YC12-2 cylinders. The singlet excitation keeps decreasing as the bond dimension $m$ increases.


FIG. 14. The $S^{z}=1$ transfer matrix spectrum of YC8-0; here, $J_{2}=0$, and the bond dimension is $m=6000$.

Therefore, it is possible that the gapless triplet excitation at certain $M$ points will be pushed to a higher energy level.

Figure 14 shows the $S^{z}=1$ transfer matrix spectrum of the cylinder YC8-0 with $J_{2}=0$. We find that the lowest modes behave like the Dirac modes. Similar to the YC2n- $(4 \mathrm{k}+2)$ cylinder, the lowest modes show a linear dependence with the twist angle $\theta$. These "Dirac modes" are actually twofold degenerate, and they have the same $k_{1}$ but distinct $k_{2}$. Since our simulation cannot adiabatically twist to the Dirac points, we cannot unambiguously determine the momentum of the Dirac points. But there are several indications that the two Dirac modes correspond to the $M_{1}=(\pi, 0)$ and $M_{3}=(\pi, \pi)$ points [labeled by $\left(k_{1}, k_{2}\right)$ ]. First, the YC8-0 cylinder has a reflection symmetry (with the reflection axis perpendicular to $\vec{a}_{1}$ ), under which $M_{1}$ transforms to $M_{3}$. Second, by doing a simple linear extrapolation for momenta $k_{1}$ [Fig. 14(b)] and $k_{2}$ [Fig. 14(c)], the momentum is consistent with $M_{1}$ and $M_{3}$. We note that the extrapolation for $k_{2}$ gives $k_{2} \approx \pm 0.1 \pi$ and $k_{2} \approx \pi \pm 0.1 \pi$, which has considerable discrepancy from $k_{2}=0$ and $k_{2}=\pi$. This discrepancy might come from the finite-size effect; for example, there is scattering (momentum transfer) between the two modes.

\section{e. Dependence of numerical data on the bond dimension}

Even in a critical system, the DMRG simulation will always produce a finite correlation length (or excitation gap) so long as the bond dimension $m$ is finite. This is the
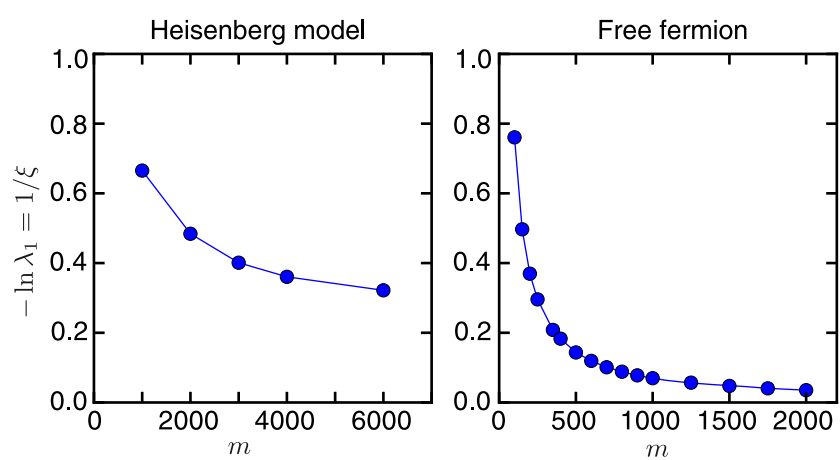

FIG. 15. The dependence of the inverse of correlation $(1 / \xi)$ at the Dirac point versus the bond dimension $m$. We show both the kagome Heisenberg model $\left(\mathrm{YC} 8-2, J_{2}=0.05\right)$ and the freefermion model.



FIG. 16. Spin gap versus the truncation error. The larger the bond dimension is, the smaller the truncation error. For the comparable truncation error, the larger system size has a smaller spin gap. Here, we show $J_{2}=0$, and the twist angle $\theta=5 \pi / 6$. The bond dimensions shown are $m=1000,2000,3000,4000$. (For YC6-2, they are $m=2000,3000,4000$.)

reason why the transfer matrix spectrum still shows a finite "gap" even at the Dirac point. One way to see that this is an artifact of the DMRG simulation is to look at the inverse of the correlation length $(1 / \xi)$ at the Dirac point versus the bond dimension $m$, as shown in Fig. 15. We see that the gap of the Dirac point decreases as the bond dimension increases.

The numerical error induced by the finite bond dimension of the DMRG also makes it difficult to compare different system sizes. For example, comparing the spin gap in Fig. 3 or the excitation spectrum of different system sizes, it appears that the gap increases with the system size. However, as far as we can tell, this is an artifact of the finite bond dimension. As we have seen before, the gap of the Dirac point becomes smaller as the numerical error (quantified via "truncation error") is decreased. On the other hand, for a larger system size, the numerical error at fixed bond dimension becomes much larger. Specifically, to achieve the same truncation error, the required bond dimension increases exponentially with the circumference of the cylinder. Figure 16 shows the spin gap 




FIG. 17. The transfer matrix spectrum of a magnetic order. Here, we show a YC8-2 cylinder, with $J_{2}=0.3$, and the bond dimension $m=2000$.

versus the truncation error, from which we see that for a comparable truncation error, the larger system size in fact has a smaller gap.

\section{f. Transfer matrix spectrum of magnetic order}

From the spectrum of the free fermions, the kagome Heisenberg model with $J_{2}=0,0.05,0.1$, one can see the trend that the yellow mode decreases by increasing $J_{2}$. We speculate that as $J_{2}$ increases, the yellow mode eventually condenses, giving rise to a magnetic order. Figure 17 shows the transfer matrix spectrum of a magnetic order at $J_{2}=0.3$, which supports the above scenario. One can see that all the lowest-lying excitation spectra are the yellow modes (no red or blue), and they have a much smaller gap than the kagome spin-liquid phase. The data shown are for a small bond dimension $m=2000$; the gap will eventually vanish as we go to the infinite bond dimension.

\section{APPENDIX C: INSTABILITY OF A DIRAC SPIN LIQUID IN THE QUASI-ONE- DIMENSIONAL LIMIT}

\section{Instability on the $\mathrm{YC} 2 \mathrm{n}-(4 \mathrm{k}+2)$ cylinder}

For the YC8-2 cylinder, once we tune the flux to $\theta=\pi$ in order to exactly hit the Dirac points, the kagome spin liquid is unstable to an ordered state. Such an ordered state breaks spin-flip symmetry $P_{x}=\prod\left(2 S^{x}\right)$ and the lattice symmetry (e.g., $T_{a_{1}}$ and $C_{6}$ ); its order pattern is shown in Fig. 18. Interestingly, such an ordered state actually preserves certain symmetries, which are (i) the reflection symmetry $R_{y}$, (ii) translational symmetry $T_{a_{2}}$, (iii) the combination of spin-flip and translational symmetry $P_{x} T_{a_{1}}$, and (iv) $X Y$ spin rotation symmetry.

One can work out the transformations for the Dirac fermions $\Psi$ [6],

$$
\begin{gathered}
T_{a_{1}}: \Psi \rightarrow\left(i \mu^{2}\right) \Psi, \\
T_{a_{2}}: \Psi \rightarrow\left(i \mu^{3}\right) \Psi, \\
P_{x}: \Psi \rightarrow\left(\sigma^{1}\right) \Psi, \\
R_{y}: \Psi \rightarrow\left(\tau^{12}\right)\left(i \mu^{12}\right) \Psi,
\end{gathered}
$$

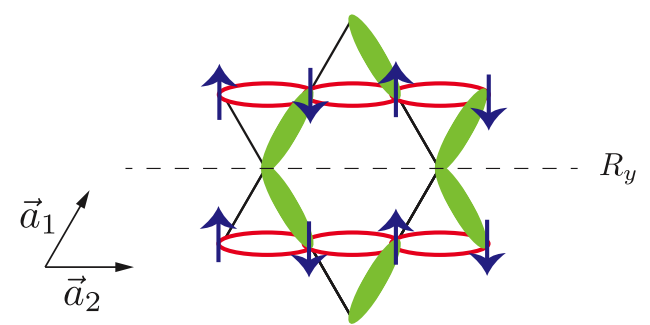

FIG. 18. The kagome spin liquid on the YC8-2 cylinder is unstable to an ordered state when $\theta=\pi$. The ordered state spontaneously breaks the spin-flip symmetry $P_{x}=\prod\left(2 S^{x}\right)$ (hence, the time-reversal symmetry) by forming a staggered Ising magnetization $\left(\left\langle S_{i}^{z}\right\rangle \approx \pm 0.03\right)$ as represented by arrows. It breaks lattice symmetry, with bond correlations $\left\langle\vec{S}_{i} \cdot \vec{S}_{j}\right\rangle$, to be -0.26 (green solid bonds), -0.22 (red hollow bonds), and -0.17 (black thin bonds).

where $\quad \tau^{12}=\left(\cos (\pi / 6) \tau^{1}-\sin (\pi / 6) \tau^{2}\right) \quad$ and $\quad \mu^{12}=$ $\left(\cos (\pi / 4) \mu^{1}-\sin (\pi / 4) \mu^{2}\right)$, and $\bar{\Psi}=i \Psi^{\dagger} \tau^{3}$. Note that $\tau$ represents the spinor index of Dirac fermions, $\sigma$ represents the spin index, and $\mu$ represents the valley index. With the above symmetry transformation rules, one can straightforwardly find the mass term (of the Dirac fermions) that gives the order pattern (Fig. 18) as $i \bar{\Psi} \sigma^{3} \mu^{3} \Psi$.

\section{Spontaneous mass generation of Dirac fermions in $1+1$ dimension}

As we discussed in Sec. IV, when the kagome spin liquid is tuned to exactly hit the Dirac points on a small cylinder [YC2n- $(4 \mathrm{k}+2)$ geometry], we numerically find an instability toward an ordered state by spontaneously generating a mass term $i \bar{\Psi} \sigma^{3} \mu^{3} \Psi$. This immediately raises the question of whether these results imply that the $U(1) \operatorname{DSL}\left(N_{f}=4\right.$ QED3) is unstable to the spontaneously chiral symmetry breaking (CSB). The CSB of QED3 is still an open issue, and it is unclear whether $N_{f}=4$ QED3 will eventually flow to an interacting conformal fixed point or not [66]. Our numerical simulation, on the other hand, was carried out on a quasi-1D cylindrical geometry, for which the issue of spontaneous mass generation is different from the 2+1D limit. The differences are twofold: (i) The $U(1)$ gauge field is more gentle in quasi-1D; it simply reduces the $N_{f}$ flavors of 1D Dirac fermions to $N_{f}-1$ coupled TomonagaLuttinger liquids (TLL) [64]. (ii) The effects of fourfermion interactions on Dirac fermions are more drastic in 1D than in in 2D; namely, in 2D, all four-fermion interactions are irrelevant for (free) Dirac fermions, while in $1 \mathrm{D}$, four-fermion interactions might be relevant or marginally relevant.

For the $U(1)$ DSL on the YC2n- $(4 \mathrm{k}+2)$ cylinder with $\theta=\pi$-flux, two Dirac fermions are gapless. Then, the dynamical $U(1)$ gauge field reduces the system to a TLL with central charge $c=1$, described by 


$$
H=\frac{v}{2}\left(\frac{1}{K}\left(\partial_{t} \phi\right)^{2}+K\left(\partial_{x} \phi\right)^{2}\right)
$$

Here, $K$ is the Luttinger parameter. Generically, there are perturbations that can potentially gap out the system. Assuming that the $U(1)$ symmetry is preserved, the most relevant perturbation is $\lambda \cos 2 \phi$, which is irrelevant when $K>1 / 2$ [67]. For $K<1 / 2$, the perturbation is relevant and will gap out the Luttinger liquid, which corresponds to the mass generation of the Dirac fermions. For $K=1 / 2$, the perturbation is marginally relevant or marginally irrelevant, depending on the sign of $\lambda$. Microscopically, it is difficult to extract the Luttinger parameter $K$. However, for the $c=1$ Luttinger liquid with $S U(2)$ symmetry, the $K$ is fixed to $K=1 / 2$. Our system is very close to this limit [the twist boundary condition slightly breaks $S U(2)$ symmetry]; hence, we expect $K \approx 1 / 2$. Consequently, it is reasonable that in our numerical simulation on a small YC2n- $(4 \mathrm{k}+2)$ (e.g., YC8-2) cylinder, the DSL is unstable to an ordered state with a mass term spontaneously generated.

[1] S. Sachdev, Kagome- and Triangular-Lattice Heisenberg Antiferromagnets: Ordering from Quantum Fluctuations and Quantum-Disordered Ground States with Unconfined Bosonic Spinons, Phys. Rev. B 45, 12377 (1992).

[2] J. T. Chalker and J. F. G. Eastmond, Ground-State Disorder in the Spin-1/2 Kagomé Heisenberg Antiferromagnet, Phys. Rev. B 46, 14201 (1992).

[3] M. B. Hastings, Dirac Structure, RVB, and Goldstone Modes in the Kagomé Antiferromagnet, Phys. Rev. B 63, 014413 (2000).

[4] R. R. P. Singh and D. A. Huse, Ground State of the Spin-1/2 Kagome-Lattice Heisenberg Antiferromagnet, Phys. Rev. B 76, 180407 (2007).

[5] Y. Ran, M. Hermele, P. A. Lee, and X.-G. Wen, ProjectedWave-Function Study of the Spin-1/2 Heisenberg Model on the Kagomé Lattice, Phys. Rev. Lett. 98, 117205 (2007).

[6] M. Hermele, Y. Ran, P. A. Lee, and X.-G. Wen, Properties of an Algebraic Spin Liquid on the Kagome Lattice, Phys. Rev. B 77, 224413 (2008).

[7] G. Evenbly and G. Vidal, Frustrated Antiferromagnets with Entanglement Renormalization: Ground State of the Spin- $-\frac{1}{2}$ Heisenberg Model on a Kagome Lattice, Phys. Rev. Lett. 104, 187203 (2010).

[8] S. Yan, D. A. Huse, and S. R. White, Spin-Liquid Ground State of the $\mathrm{S}=1 / 2$ Kagome Heisenberg Antiferromagnet, Science 332, 1173 (2011).

[9] A. M. Läuchli, J. Sudan, and E. S. Sørensen, Ground-State Energy and Spin Gap of Spin- $\frac{1}{2}$ Kagomé-Heisenberg Antiferromagnetic Clusters: Large-Scale Exact Diagonalization Results, Phys. Rev. B 83, 212401 (2011).

[10] H. Nakano and T. Sakai, Numerical-Diagonalization Study of Spin Gap Issue of the Kagome Lattice Heisenberg Antiferromagnet, J. Phys. Soc. Jpn. 80, 053704 (2011).
[11] S. Depenbrock, I. P. McCulloch, and U. Schollwöck, Nature of the Spin-Liquid Ground State of the s = 1/2 Heisenberg Model on the Kagome Lattice, Phys. Rev. Lett. 109, 067201 (2012).

[12] H.-C. Jiang, Z. Wang, and L. Balents, Identifying Topological Order by Entanglement Entropy, Nat. Phys. 8, 902 (2012).

[13] L. Messio, B. Bernu, and C. Lhuillier, Kagome Antiferromagnet: A Chiral Topological Spin Liquid?, Phys. Rev. Lett. 108, 207204 (2012).

[14] Y. Iqbal, F. Becca, and D. Poilblanc, Projected Wave Function Study of $\mathbb{Z}_{2}$ Spin Liquids on the Kagome Lattice for the Spin- $\frac{1}{2}$ Quantum Heisenberg Antiferromagnet, Phys. Rev. B 84, 020407 (2011).

[15] Y. Iqbal, F. Becca, S. Sorella, and D. Poilblanc, Gapless Spin-Liquid Phase in the Kagome Spin- $\frac{1}{2}$ Heisenberg Antiferromagnet, Phys. Rev. B 87, 060405 (2013).

[16] Y. Iqbal, D. Poilblanc, and F. Becca, Vanishing Spin Gap in a Competing Spin-Liquid Phase in the Kagome Heisenberg Antiferromagnet, Phys. Rev. B 89, 020407 (2014).

[17] Y. Iqbal, D. Poilblanc, and F. Becca, Spin- $\frac{1}{2}$ Heisenberg $J_{1}-J_{2}$ Antiferromagnet on the Kagome Lattice, Phys. Rev. B 91, 020402 (2015).

[18] S. Nishimoto, N. Shibata, and C. Hotta, Controlling Frustrated Liquids and Solids with an Applied Field in a Kagome Heisenberg Antiferromagnet, Nat. Commun. 4, 2287 (2013).

[19] Y.-C. He and Y. Chen, Distinct Spin Liquids and Their Transitions in Spin-1/2 xxz. Kagome Antiferromagnets, Phys. Rev. Lett. 114, 037201 (2015).

[20] A. M. Läuchli and R. Moessner, Quantum Simulations Made Easy Plane, arXiv:1504.04380.

[21] J.-W. Mei, J.-Y. Chen, H. He, and X.-G. Wen, Gapped Spin Liquid with $\mathbb{Z}$ 2-Topological Order for Kagome Heisenberg Model, Phys. Rev. B 95, 235107 (2017).

[22] S. Jiang, P. Kim, J. H. Han, and Y. Ran, Competing Spin Liquid Phases in the $\mathrm{S}=\frac{1}{2}$ Heisenberg Model on the Kagome Lattice, arXiv:1610.02024.

[23] H. J. Liao, Z. Y. Xie, J. Chen, Z. Y. Liu, H.D. Xie, R. Z. Huang, B. Normand, and T. Xiang, Gapless SpinLiquid Ground State in the $s=1 / 2$ Kagome Antiferromagnet, Phys. Rev. Lett. 118, 137202 (2017).

[24] A. M. Läuchli, J. Sudan, and R. Moessner, The $S=1 / 2$ Kagome Heisenberg Antiferromagnet Revisited, arXiv:1611 .06990 .

[25] J. S. Helton, K. Matan, M. P. Shores, E. A. Nytko, B. M. Bartlett, Y. Yoshida, Y. Takano, A. Suslov, Y. Qiu, J.-H. Chung, D. G. Nocera, and Y.S. Lee, Spin Dynamics of the Spin-1/2 Kagome Lattice Antiferromagnet $\mathrm{ZnCu}_{3}(\mathrm{OH})_{6} \mathrm{Cl}_{2}$, Phys. Rev. Lett. 98, 107204 (2007).

[26] T.-H. Han, J. S. Helton, S. Chu, D. G. Nocera, J. A. Rodriguez-Rivera, C. Broholm, and Y. S. Lee, Fractionalized Excitations in the Spin-Liquid State of a KagomeLattice Antiferromagnet, Nature (London) 492, 406 (2012).

[27] P. W. Anderson, Resonating Valence Bonds: A New Kind of Insulator?, Mater. Res. Bull. 8, 153 (1973).

[28] L. Savary and L. Balents, Quantum Spin Liquids, Rep. Prog. Phys. 80, 016502 (2017).

[29] Y. Zhou, K. Kanoda, and T.-K. Ng, Quantum Spin Liquid States, Rev. Mod. Phys. 89, 025003 (2017). 
[30] N. Read and B. Chakraborty, Statistics of the Excitations of the Resonating-Valence-Bond State, Phys. Rev. B 40, 7133 (1989).

[31] X. G. Wen, Mean-Field Theory of Spin-Liquid States with Finite Energy Gap and Topological Orders, Phys. Rev. B 44, 2664 (1991).

[32] R. Moessner and S. L. Sondhi, Resonating Valence Bond Phase in the Triangular Lattice Quantum Dimer Model, Phys. Rev. Lett. 86, 1881 (2001).

[33] X.-G. Wen, Topological Orders in Rigid States, Int. J. Mod. Phys. B 04, 239 (1990).

[34] Y. Zhang, T. Grover, A. Turner, M. Oshikawa, and A. Vishwanath, Quasiparticle Statistics and Braiding from Ground-State Entanglement, Phys. Rev. B 85, 235151 (2012).

[35] L. Cincio and G. Vidal, Characterizing Topological Order by Studying the Ground States on an Infinite Cylinder, Phys. Rev. Lett. 110, 067208 (2013).

[36] M. P. Zaletel, R. S. K. Mong, and F. Pollmann, Topological Characterization of Fractional Quantum Hall Ground States from Microscopic Hamiltonians, Phys. Rev. Lett. 110, 236801 (2013).

[37] Y.-C. He, D. N. Sheng, and Y. Chen, Chiral Spin Liquid in a Frustrated Anisotropic Kagome Heisenberg Model, Phys. Rev. Lett. 112, 137202 (2014).

[38] S.-S. Gong, W. Zhu, and D. N. Sheng, Emergent Chiral Spin Liquid: Fractional Quantum Hall Effect in a Kagome Heisenberg Model, Sci. Rep. 4, 6317 (2014).

[39] M. Barkeshli, Transitions Between Chiral Spin Liquids and Z2 Spin Liquids, arXiv:1307.8194.

[40] M. P. Zaletel and A. Vishwanath, Constraints on Topological Order in Mott Insulators, Phys. Rev. Lett. 114, 077201 (2015).

[41] Y.-C. He, Y. Fuji, and S. Bhattacharjee, Kagome Spin Liquid: A Deconfined Critical Phase Driven by U(1) Gauge Fluctuation, arXiv:1512.05381.

[42] P. Nikolić and T. Senthil, Theory of the Kagome Lattice Ising Antiferromagnet in Weak Transverse Fields, Phys. Rev. B 71, 024401 (2005).

[43] Y.-C. He, S. Bhattacharjee, F. Pollmann, and R. Moessner, Kagome Chiral Spin Liquid as a Gauged U(1) Symmetry Protected Topological Phase, Phys. Rev. Lett. 115, 267209 (2015).

[44] M. Fu, T. Imai, T.-H. Han, and Y. S. Lee, Evidence for a Gapped Spin-Liquid Ground State in a Kagome Heisenberg Antiferromagnet, Science 350, 655 (2015).

[45] S. R. White, Density Matrix Formulation for Quantum Renormalization Groups, Phys. Rev. Lett. 69, 2863 (1992).

[46] S. R. White, Density-Matrix Algorithms for Quantum Renormalization Groups, Phys. Rev. B 48, 10345 (1993).

[47] I. P. McCulloch, Infinite Size Density Matrix Renormalization Group and Revisited, arXiv:0804.2509.

[48] V. Zauner, D. Draxler, L. Vanderstraeten, M. Degroote, J. Haegeman, M. M. Rams, V. Stojevic, N. Schuch, and F. Verstraete, Transfer Matrices and Excitations with Matrix Product States, New J. Phys. 17, 053002 (2015).

[49] Ch. Waldtmann, H.-U. Everts, B. Bernu, C. Lhuillier, P. Sindzingre, P. Lecheminant, and L. Pierre, First Excitations of the Spin 1/2 Heisenberg Antiferromagnet on the Kagomé Lattice, Eur. Phys. J. B 2, 501 (1998).
[50] X.-G. Wen, Quantum Orders and Symmetric Spin Liquids, Phys. Rev. B 65, 165113 (2002).

[51] F. Wang and A. Vishwanath, Spin-Liquid States on the Triangular and Kagomé Lattices: A Projective-SymmetryGroup Analysis of Schwinger Boson States, Phys. Rev. B 74, 174423 (2006).

[52] Y.-M. Lu, Y. Ran, and P. A. Lee, $\mathbb{Z}_{2}$ Spin Liquids in the $s=\frac{1}{2}$ Heisenberg Model on the Kagome Lattice: A Projective Symmetry-Group Study of Schwinger Fermion Mean-Field States, Phys. Rev. B 83, 224413 (2011).

[53] Y. Qi and M. Cheng, Classification of Symmetry Fractionalization in Gapped $\mathbb{Z}_{2}$ Spin Liquids, arXiv:1606.04544.

[54] Y.-M. Lu, Symmetry Protected Gapless $Z_{2}$ Spin Liquids, Phys. Rev. B 93, 165113 (2016).

[55] A. Wietek, A. Sterdyniak, and A. M. Läuchli, Nature of Chiral Spin Liquids on the Kagome Lattice, Phys. Rev. B 92, 125122 (2015).

[56] W.-J. Hu, W. Zhu, Y. Zhang, S. Gong, F. Becca, and D. N. Sheng, Variational Monte Carlo Study of a Chiral Spin Liquid in the Extended Heisenberg Model on the Kagome Lattice, Phys. Rev. B 91, 041124 (2015).

[57] X. G. Wen and Q. Niu, Ground-State Degeneracy of the Fractional Quantum Hall States in the Presence of a Random Potential and on High-Genus Riemann Surfaces, Phys. Rev. B 41, 9377 (1990).

[58] R. B. Laughlin, Quantized Hall Conductivity in Two Dimensions, Phys. Rev. B 23, 5632 (1981).

[59] M. Oshikawa, Commensurability, Excitation Gap, and Topology in Quantum Many-Particle Systems on a Periodic Lattice, Phys. Rev. Lett. 84, 1535 (2000).

[60] Y.-C. He, D. N. Sheng, and Y. Chen, Obtaining Topological Degenerate Ground States by the Density Matrix Renormalization Group, Phys. Rev. B 89, 075110 (2014).

[61] U. Schollwöck, The Density-Matrix Renormalization Group in the Age of Matrix Product States, Ann. Phys. (Amsterdam) 326, 96 (2011).

[62] J. Haegeman, V. Zauner, N. Schuch, and F. Verstraete, Shadows of Anyons and the Entanglement Structure of Topological Phases, Nat. Commun. 6, 8284 (2015).

[63] M. Hermele, T. Senthil, and M. P. A. Fisher, Algebraic Spin Liquid as the Mother of Many Competing Orders, Phys. Rev. B 72, 104404 (2005); Phys. Rev. B 76, 149906(E) (2007).

[64] D. N. Sheng, O. I. Motrunich, and M. P. A. Fisher, Spin Bose-Metal Phase in a Spin- $\frac{1}{2}$ Model with Ring Exchange on a Two-Leg Triangular Strip, Phys. Rev. B 79, 205112 (2009).

[65] B. Bauer, L. Cincio, B. P. Keller, M. Dolfi, G. Vidal, S. Trebst, and A. W. W. Ludwig, Chiral Spin Liquid and Emergent Anyons in a Kagome Lattice Mott Insulator, Nat. Commun. 5, 5137 (2014).

[66] T. Grover, Entanglement Monotonicity and the Stability of Gauge Theories in Three Spacetime Dimensions, Phys. Rev. Lett. 112, 151601 (2014).

[67] Thierry Giamarchi, Quantum Physics in One Dimension, International Series of Monographs on Physics (Clarendon Press, Oxford, 2004). 